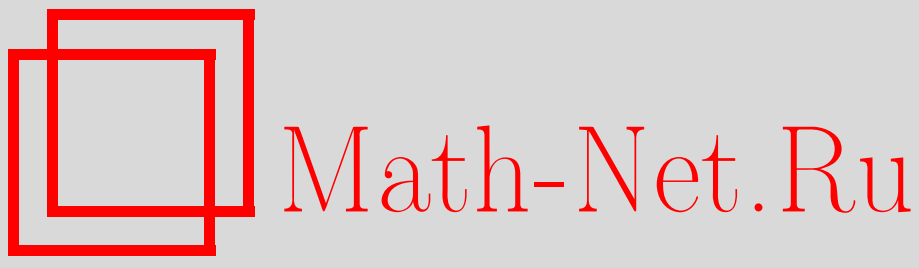

Г. Л. Харатишвили, Т. А. Тадумадзе, Формулы вариации решения дифференциального уравнения с запаздывающими аргументами и разрывным начальным условием, Матем. сб., 2005, том 196, номер 8, 49-74

DOI: https://doi.org/10.4213/sm1406

Использование Общероссийского математического портала Math-Net.Ru подразумевает, что вы прочитали и согласны с пользовательским соглашением http://www. mathnet.ru/rus/agreement

Параметры загрузки:

IP : 54.209 .52 .79

26 апреля 2023 г., $18: 31: 14$ 
УДК 517.929

\author{
Г. Л. Харатишвили, Т.А. Тадумадзе
}

\title{
Формулы вариации решения дифференциального уравнения с запаздывающими аргументами и разрывным начальным условием
}

\begin{abstract}
В работе доказаны формулы вариации решения для нелинейного дифференциального уравнения с переменньми запаздываниями и разрьвным начальньпм условием. Разрьвность начального условия означает, что в начальный момент времени значения начальной функции и траектории, вообще говоря, не совпадают. Полученные формулы содержат новое слагаемое, связанное с разрывностью начального условия и вариацией начального момента.

Библиограбиия: 11 названий.
\end{abstract}

Формулы вариации решения дифференциального уравнения относительно правой части уравнения и начальных данных играют важную роль при доказательстве необходимых условий оптимальности для задач оптимального управления [1]-[8]. Кроме того, они дают возможность получить аналитический вид приближенного решения возмушенного дифференциального уравнения с возмушенными начальными условиями.

В предлагаемой работе для нелинейного дифференциального уравнения с переменными запаздываниями и разрывным начальньп условием доказаны формулы вариации решения в окрестности правого конца основного интервала $\left[\tilde{t}_{0}, \widetilde{t}_{1}\right]$ при возмущении начального момента $\widetilde{t}_{0}$, правой части уравнения, начальной функции и начального значения решения.

Формулы вариации решения хорошо известны: для обыкновенных дифференциальных уравнений см. [6], [8], [9], в том числе для уравнений с запаздываниями см. [4], [5], [7]; для функционально-дифференциальных уравнений см. [4], [10]. Однако в [5], [7], [10] не рассматривается вариация начального момента, а в [4], [6], [8], [9] - разрывность начального условия.

В $\S 1$ настоящей работы сформулированы основные теоремы, учитывающие левостороннюю, правостороннюю и двустороннюю вариации начального момента $\widetilde{t}_{0}$. Эти теоремы обобщают результаты работ [5], [7], [11].

Доказательства основных теорем даны в $\S \S 3,4$.

В $\S 2$ для одного класса функционально-дифференциальных уравнений установлен порядок прирашения решения относительно малого параметра и получено аналитическое выражение прирашения решения в начальный момент $\widetilde{t}_{0}$. Эти результаты существенно используются при доказательстве основных теорем.

(C) Г. Л. ХАРАтИшвили, Т. А. ТАдУМАдЗЕ 2005 


\section{§1. Формулировка основных результатов}

Пусть $J=[a, b]$ - конечный интервал, $O \subset \mathbb{R}^{n}$ - открытое множество, $E$ множество функций $f: J \times O^{s} \rightarrow \mathbb{R}^{n}$, удовлетворяющих следуюшим условиям: для почти всех $t \in J$ функция $f(t, \cdot): O^{s} \rightarrow \mathbb{R}^{n}$ непрерывно дифференцируема; при каждом $\left(x_{1}, \ldots, x_{s}\right) \in O^{s}$ функции $f\left(t, x_{1}, \ldots, x_{s}\right), f_{x_{i}}\left(t, x_{1}, \ldots, x_{s}\right), i=1, \ldots, s$, измеримы на $J$; для любой $f \in E$ и любого компакта $K \subset O$ существует такая функция $m_{f, K}(\cdot) \in L\left(J, \mathbb{R}_{+}\right), \mathbb{R}_{+}=[0, \infty)$, что при любом $\left(x_{1}, \ldots, x_{s}\right) \in K^{s}$ и для почти всех $t \in J$

$$
\left|f\left(t, x_{1}, \ldots, x_{s}\right)\right|+\sum_{i=1}^{s}\left|f_{x_{i}}\left(t, x_{1}, \ldots, x_{s}\right)\right| \leqslant m_{f, K}(t) .
$$

Пусть, далее, скалярные функции $\tau_{i}(t), t \in \mathbb{R}, i=1, \ldots, s$, абсолютно непрерывны и удовлетворяют условиям: $\tau_{i}(t) \leqslant t, \dot{\tau}_{i}(t)>0, i=1, \ldots, s ; \Phi$-множество непрерывных функций $\varphi: J_{1}=\left[\tau_{0}, b\right] \rightarrow O, \tau_{0}=\min \left(\tau_{1}(a), \ldots, \tau_{s}(a)\right)$, при этом $\|\varphi\|=\sup \left\{|\varphi(t)|: t \in J_{1}\right\}$.

Каждому элементу $\mu=\left(t_{0}, x_{0}, \varphi, f\right) \in A=J \times O \times \Phi \times E$ будем ставить в соответствие дифференциальное уравнение

$$
\dot{x}(t)=f\left(t, x\left(\tau_{1}(t)\right), \ldots, x\left(\tau_{s}(t)\right)\right)
$$

с разрывным начальньм условием

$$
x(t)=\varphi(t), \quad t \in\left[\tau_{0}, t_{0}\right), \quad x\left(t_{0}\right)=x_{0} .
$$

ОПРЕДЕЛЕНИЕ 1.1. Пусть $\mu=\left(t_{0}, x_{0}, \varphi, f\right) \in A, t_{0}<b$. Функцию $x(t, \mu) \in O$, $t \in\left[\tau_{0}, t_{1}\right], t_{1} \in\left(t_{0}, b\right]$, будем назьвать решением уравнения (1.1) с начальным условием (1.2) или решением, соответствующим әлементу $\mu$ и определенным на интервале $\left[\tau_{0}, t_{1}\right]$, если она на интервале $\left[\tau_{0}, t_{0}\right]$ удовлетворяет условию $(1.2)$, а на интервале $\left[t_{0}, t_{1}\right]$ абсолютно непрерьвна и почти всюду (п.в.) на $\left[t_{0}, t_{1}\right]$ удовлетворяет уравнению (1.1).

Введем множество вариаций:

$$
\begin{aligned}
V= & \left\{\delta \mu=\left(\delta t_{0}, \delta x_{0}, \delta \varphi, \delta f\right) \in \mathbb{R} \times \mathbb{R}^{n} \times(\Phi-\widetilde{\varphi}) \times E:\right. \\
& \left.\left|\delta t_{0}\right| \leqslant \alpha,\left|\delta x_{0}\right| \leqslant \alpha,\|\delta \varphi\| \leqslant \alpha, \delta f=\sum_{i=1}^{k} \lambda_{i} \delta f_{i},\left|\lambda_{i}\right| \leqslant \alpha, i=1, \ldots, k\right\},
\end{aligned}
$$

где $\delta f_{i} \in E, i=1, \ldots, k,-$ фиксированные точки, $\widetilde{\varphi} \in \Phi$ - фиксированная точка, $\alpha>0$ - фиксированное число.

Пусть $\widetilde{x}(t)$ - соответствуюшее элементу $\widetilde{\mu}=\left(\widetilde{t}_{0}, \widetilde{x}_{0}, \widetilde{\varphi}, \widetilde{f}\right) \in A$ решение, определенное на интервале $\left[\tau_{0}, \widetilde{t}_{1}\right], \widetilde{t}_{i} \in(a, b), i=0,1$. Существуют такие числа $\varepsilon_{1}>0$, $\delta_{1}>0$, что для произвольного $(\varepsilon, \delta \mu) \in\left[0, \varepsilon_{1}\right] \times V$ элемент $\widetilde{\mu}+\varepsilon \delta \mu \in A$ и ему 
соответствует решение $x(t, \widetilde{\mu}+\varepsilon \delta \mu)$, определенное на интервале $\left[\tau_{0}, \widetilde{t}_{1}+\delta_{1}\right] \subset J_{1}$ (см. лемму 2.2).

В силу единственности решение $x(t, \widetilde{\mu})$ на интервале $\left[\tau_{0}, \widetilde{t}_{1}+\delta_{1}\right]$ является продолжением решения $\widetilde{x}(t)$. Поэтому в дальнейшем мы можем считать, что решение $\widetilde{x}(t)$ с самого начала определено на всем интервале $\left[\tau_{0}, \widetilde{t}_{1}+\delta_{1}\right]$.

По определению прирашение решения $\widetilde{x}(t)=x(t, \widetilde{\mu})$ есть

$\Delta x(t)=\Delta x(t, \varepsilon \delta \mu)=x(t, \widetilde{\mu}+\varepsilon \delta \mu)-\widetilde{x}(t), \quad(t, \varepsilon, \delta \mu) \in\left[\tau_{0}, \widetilde{t}_{1}+\delta_{1}\right] \times\left[0, \varepsilon_{1}\right] \times V$.

Для формулировки основных результатов введем следующие обозначения:

$$
\sigma_{i}=(\widetilde{t}_{0}, \underbrace{\widetilde{x}_{0}, \ldots, \widetilde{x}_{0}}_{i}, \underbrace{\widetilde{\varphi}\left(\widetilde{t_{0}}\right), \ldots, \widetilde{\varphi}\left(\widetilde{t_{0}}\right)}_{(p-i)}, \widetilde{\varphi}\left(\tau_{p+1}\left(\widetilde{t}_{0}\right)\right), \ldots, \widetilde{\varphi}\left(\tau_{s}\left(\widetilde{t}_{0}\right)\right)), \quad i=0, \ldots, p
$$

(роль числа $p$ будет выясняться ниже); если $i=0$, то $\sigma_{0}$ не содержит $\widetilde{x}_{0}$; если $i=p$, то $\sigma_{p}$ не содержит $\widetilde{\varphi}\left(\widetilde{t}_{0}\right)$; далее, $\gamma_{i}^{0}=\gamma_{i}\left(\widetilde{t_{0}}\right), \gamma_{i}(t)=\tau_{i}^{-1}(t)$;

$$
\begin{gathered}
\sigma_{i}=\left(\gamma_{i}^{0}, \widetilde{x}\left(\tau_{1}\left(\gamma_{i}^{0}\right)\right), \ldots, \widetilde{x}\left(\tau_{i-1}\left(\gamma_{i}^{0}\right)\right), \widetilde{x} 0, \widetilde{\varphi}\left(\tau_{i+1}\left(\gamma_{i}^{0}\right)\right), \ldots, \widetilde{\varphi}\left(\tau_{s}\left(\gamma_{i}^{0}\right)\right)\right), \\
\quad i=p+1, \ldots, s \\
\sigma_{i}^{0}=\left(\gamma_{i}^{0}, \widetilde{x}\left(\tau_{1}\left(\gamma_{i}^{0}\right)\right), \ldots, \widetilde{x}\left(\tau_{i-1}\left(\gamma_{i}^{0}\right)\right), \widetilde{\varphi}\left(\widetilde{t_{0}}\right), \widetilde{\varphi}\left(\tau_{i+1}\left(\gamma_{i}^{0}\right)\right), \ldots, \widetilde{\varphi}\left(\tau_{i}\left(\gamma_{i}^{0}\right)\right)\right), \\
\quad i=p+1, \ldots, s
\end{gathered}
$$

ТЕОРема 1.1. Пусть выполнены условия:

1.1) $\gamma_{i}^{0}=\widetilde{t}_{0}, i=1, \ldots, p, \gamma_{p+1}^{0}<\cdots<\gamma_{s}^{0}<\widetilde{t}_{1}$;

1.2) существует такое число $\delta>0$, ито

$$
\gamma_{1}(t) \leqslant \cdots \leqslant \gamma_{p}(t), \quad t \in\left(\widetilde{t_{0}}-\delta, \widetilde{t}_{0}\right]
$$

1.3) существуют конечные предель

$$
\begin{gathered}
\dot{\gamma}_{i}^{-}=\dot{\gamma}_{i}\left(\widetilde{t}_{0}-\right), \quad i=1, \ldots, s ; \\
\lim _{\omega \rightarrow \sigma_{i}} \widetilde{f}(\omega)=f_{i}^{-}, \quad \omega=\left(t, x_{1}, \ldots, x_{s}\right) \in\left(\widetilde{t_{0}}-\delta, \widetilde{t}_{0}\right] \times O^{s}, \quad i=0, \ldots, p ; \\
\lim _{\left(\omega_{1}, \omega_{2}\right) \rightarrow\left(\sigma_{i}, \sigma_{i}^{0}\right)}\left(\widetilde{f}\left(\omega_{1}\right)-\widetilde{f}\left(\omega_{2}\right)\right)=f_{i}^{-}, \\
\left(\omega_{1}, \omega_{2}\right) \in\left(\gamma_{i}^{0}-\delta, \gamma_{i}^{0}\right] \times O^{s}, \quad i=p+1, \ldots, s .
\end{gathered}
$$

Тогда существуют такие числа $\varepsilon_{2}>0, \delta_{2}>0$, что для произвольного

$$
(t, \varepsilon, \delta \mu) \in\left[\widetilde{t}_{1}-\delta_{2}, \widetilde{t}_{1}+\delta_{2}\right] \times\left[0, \varepsilon_{2}\right] \times V^{-}, \quad V^{-}=\left\{\delta \mu \in V: \delta t_{0} \leqslant 0\right\}
$$


справедлива формула

$$
\Delta x(t, \varepsilon \delta \mu)=\varepsilon \delta x(t, \delta \mu)+o(t, \varepsilon \delta \mu)^{1}
$$

$2 \partial e$

$$
\begin{gathered}
\delta x(t, \delta \mu)=\left(Y\left(\widetilde{t}_{0}, t\right) \sum_{i=0}^{p}\left(\widehat{\gamma}_{i+1}^{-}-\widehat{\gamma}_{i}^{-}\right) f_{i}^{-}-\sum_{i=p+1}^{s} Y\left(\gamma_{i}^{0}, t\right) f_{i}^{-} \dot{\gamma}_{i}^{-}\right) \delta t_{0}+\beta(t, \delta \mu) \\
\widehat{\gamma}_{0}^{-}=1, \quad \widehat{\gamma}_{i}^{-}=\dot{\gamma}_{i}^{-}, \quad i=1, \ldots, p, \quad \widehat{\gamma}_{p+1}^{-}=0 ; \\
\beta(t, \delta \mu)=Y\left(\widetilde{t}_{0}, t\right) \delta x_{0}+\sum_{i=p+1}^{s} \int_{\tau_{i}\left(\widetilde{t}_{0}\right)}^{\tilde{t}_{0}} Y\left(\gamma_{i}(\xi), t\right) \widetilde{f}_{x_{i}}\left[\gamma_{i}(\xi)\right] \dot{\gamma}_{i}(\xi) \delta \varphi(\xi) d \xi \\
\quad+\int_{\widetilde{t}_{0}}^{t} Y(\xi, t) \delta f[\xi] d \xi, \\
\widetilde{f}_{x_{i}}[\xi]=\widetilde{f}_{x_{i}}\left(\xi, \widetilde{x}\left(\tau_{1}(\xi)\right), \ldots, \widetilde{x}\left(\tau_{s}(\xi)\right)\right), \quad \delta f[\xi]=\delta f\left(\xi, \widetilde{x}\left(\tau_{1}(\xi)\right), \ldots, \widetilde{x}\left(\tau_{s}(\xi)\right)\right) ;
\end{gathered}
$$

$Y(\xi, t)$ - матричная функиия, удовлетворяющая уравнению

$$
\frac{\partial Y(\xi, t)}{\partial \xi}=-\sum_{i=1}^{s} Y\left(\gamma_{i}(\xi), t\right) \tilde{f}_{x_{i}}\left[\gamma_{i}(\xi)\right] \dot{\gamma}_{i}(\xi), \quad \xi \in\left[\widetilde{t}_{0}, t\right]
$$

и условию

$$
Y(\xi, t)= \begin{cases}I, & \xi=t \\ \Theta, & \xi>t\end{cases}
$$

əде I - единичная матрица, $\Theta$ - нулевая матрица.

Функция $\delta x(t, \delta \mu)$ называется вариацией решения $\widetilde{x}(t), t \in\left[\widetilde{t}_{1}-\delta_{2}, \widetilde{t}_{1}+\delta_{2}\right]$, а выражение (1.6) - формулой вариации.

ТЕОРЕМА 1.2. Пусть выполнень условие 1.1) и нижеследующие условия:

1.4) существует такое число $\delta>0$, что

$$
\gamma_{1}(t) \leqslant \cdots \leqslant \gamma_{p}(t), \quad t \in\left(\tilde{t}_{0}, \tilde{t}_{0}+\delta\right]
$$

1.5) существуют конечные предельи

$$
\begin{aligned}
& \dot{\gamma}_{i}^{+}=\dot{\gamma}_{i}\left(\widetilde{t}_{0}+\right), \quad i=1, \ldots, s \\
& \lim _{\omega \rightarrow \sigma_{i}} \tilde{f}(\omega)=f_{i}^{+}, \quad \omega=\left(t, x_{1}, \ldots, x_{s}\right) \in\left(\tilde{t}_{0}, \widetilde{t}_{0}+\delta\right] \times O^{s}, \quad i=0, \ldots, p \\
& \lim _{\left(\omega_{1}, \omega_{2}\right) \rightarrow\left(\sigma_{i}, \sigma_{i}^{0}\right)}\left(\tilde{f}\left(\omega_{1}\right)-\tilde{f}\left(\omega_{2}\right)\right)=f_{i}^{+}, \\
& \left(\omega_{1}, \omega_{2}\right) \in\left[\gamma_{i}^{0}, \gamma_{i}^{0}+\delta\right] \times O^{s}, \quad i=p+1, \ldots, s .
\end{aligned}
$$

\footnotetext{
${ }^{1}$ Здесь и далее величины (скалярные и векторные), имеющие равномерно относительно $(t, \delta \mu)$ соответствующий порядок малости по $\varepsilon$, обозначим через $O(t, \varepsilon \delta \mu), o(t, \varepsilon \delta \mu)$.
} 
Тогда существуют такие числа $\varepsilon_{2}>0, \delta_{2}>0$, что для произвольного

$$
(t, \varepsilon, \delta \mu) \in\left[\widetilde{t}_{1}-\delta_{2}, \widetilde{t}_{1}+\delta_{2}\right] \times\left[0, \varepsilon_{2}\right] \times V^{+}, \quad V^{+}=\left\{\delta \mu \in V: \delta t_{0} \geqslant 0\right\}
$$

справедлива формула $(1.5)$, әде $\delta x(t, \delta \mu)$ имеет вид

$$
\begin{gathered}
\delta x(t, \delta \mu)=\left(Y\left(\tilde{t}_{0}, t\right) \sum_{i=0}^{p}\left(\widehat{\gamma}_{i+1}^{+}-\widehat{\gamma}_{i}^{+}\right) f_{i}^{+}-\sum_{i=p+1}^{s} Y\left(\gamma_{i}^{0}, t\right) f_{i}^{+} \dot{\gamma}_{i}^{+}\right) \delta t_{0}+\beta(t, \delta \mu), \\
\widehat{\gamma}_{0}^{+}=1, \quad \widehat{\gamma}_{i}^{+}=\dot{\gamma}_{i}^{+}, \quad i=1, \ldots, p, \quad \widehat{\gamma}_{p+1}^{+}=0 .
\end{gathered}
$$

Нижеприведенная теорема является следствием теорем 1.1 и 1.2.

ТЕОРема 1.3. Пусть выполнены условия теорем 1.1 и 1.2. Пусть, кроме того, выполнены равенства

$$
\begin{gathered}
\sum_{i=0}^{p}\left(\widehat{\gamma}_{i+1}^{-}-\widehat{\gamma}_{i}^{-}\right) f_{i}^{-}=\sum_{i=0}^{p}\left(\widehat{\gamma}_{i+1}^{+}-\widehat{\gamma}_{i}^{+}\right) f_{i}^{+}=f_{0}, \\
f_{i}^{-} \dot{\gamma}_{i}^{-}=f_{i}^{+} \dot{\gamma}_{i}^{+}=f_{i}, \quad i=p+1, \ldots, s .
\end{gathered}
$$

Тогда существуют такие числа $\varepsilon_{2}>0, \delta_{2}>0$, что для произвольного

$$
(t, \varepsilon, \delta \mu) \in\left[\widetilde{t}_{1}-\delta_{2}, \widetilde{t}_{1}+\delta_{2}\right] \times\left[0, \varepsilon_{2}\right] \times V
$$

справедлива формула $(1.5)$, әде $\delta x(t, \delta \mu)$ имеет вид

$$
\delta x(t, \delta \mu)=\left(Y\left(\widetilde{t_{0}}, t\right) f_{0}-\sum_{i=p+1}^{s} Y\left(\gamma_{i}^{0}, t\right) f_{i}\right) \delta t_{0}+\beta(t, \delta \mu) .
$$

НЕкотоРЫЕ ЗАМЕЧАНИЯ. 1) Пусть $\widetilde{\varphi}\left(\widetilde{t}_{0}\right)=\widetilde{x}_{0}$, тогда $\sigma_{0}=\cdots=\sigma_{p}, \sigma_{i}=\sigma_{i}^{0}$, $i=p+1, \ldots, s$. Поэтому

$$
f_{0}^{-}=\cdots=f_{p}^{-}, \quad f_{i}^{-}=0, \quad i=p+1, \ldots, s .
$$

В этом случае формула (1.6) принимает вид

$$
\delta x(t, \delta \mu)=-Y\left(\widetilde{t_{0}}, t\right) f_{0}^{-}+\beta(t, \delta \mu) .
$$

2) Пусть $\gamma_{i}^{0}>\widetilde{t}_{0}, i=1, \ldots, s$, тогда в формуле (1.6) предполагается, что $p=0$. В этом случае условие 1.2 ) становится лишним.

3) Условие 1.2) выполнено, если $\dot{\gamma}_{p}^{-}<\cdots<\dot{\gamma}_{1}^{-}$.

В самом деле,

$$
\gamma_{i}(t)-\gamma_{i+1}(t)=\int_{\widetilde{t}_{0}}^{t}\left(\gamma_{i}(\xi)-\gamma_{i+1}(\xi)\right) d \xi=\left(\dot{\gamma}_{i}^{-}-\dot{\gamma}_{i+1}^{-}\right)\left(t-\widetilde{t}_{0}\right)+o\left(\left|t-\widetilde{t}_{0}\right|\right)
$$

Очевидно, что при достаточно малом $\delta>0$ из последнего равенства следуют неравенства

$$
\gamma_{i}(t) \leqslant \gamma_{i+1}(t), \quad i=1, \ldots, p-1, \quad t \in\left(\widetilde{t}_{0}-\delta, \widetilde{t}_{0}\right]
$$

Аналогично можно доказать, что если $\dot{\gamma}_{1}^{+}<\cdots<\dot{\gamma}_{p}^{+}$, то выполнено условие 1.4$)$. 
4) Очевидно, что все предпосылки теоремы 1.3 выполнены, если функция $\tilde{f} \in E$ непрерывна в точках $\sigma_{i}, i=0, \ldots, s$, и $\sigma_{i}^{0}, i=p+1, \ldots, s$, а функции $\dot{\gamma}_{i}(t), i=$ $1, \ldots, s$, непрерьвны в точке $\widetilde{t}_{0}$. Ясно, что в этом случае $\dot{\gamma}_{i}\left(\widetilde{t}_{0}\right)=1, i=1, \ldots, p$ (см. условие 1.1)),

$$
\begin{gathered}
f_{0}=-\widetilde{f}\left(\sigma_{p}\right), \\
f_{i}=\dot{\gamma}_{i}\left(\widetilde{t_{0}}\right)\left(\widetilde{f}\left(\sigma_{i}\right)-\widetilde{f}\left(\sigma_{i}^{0}\right)\right), \quad i=p+1, \ldots, s .
\end{gathered}
$$

5) Формулы вариации решения для дифференциального уравнения с одним запаздыванием и разрывным начальным условием при возмушении начальных данных и правой части уравнения доказаны в [11].

6) Доказанные формулы вариации непосредственно используются при доказательстве необходимых условий оптимальности для нелинейной управляемой дифференциальной системы с запаздываниями, нефиксированньм начальным моментом и разрывным начальным условием.

\section{$\S 2$. Леммы об оценке приращения решения}

Каждому элементу $\mu=\left(t_{0}, x_{0}, \varphi, f\right) \in A$ сопоставим функционально-дифференциальное уравнение

$$
\dot{y}(t)=f\left(t_{0}, \varphi, y\right)(t)=f\left(t, h\left(t_{0}, \varphi, y\right)\left(\tau_{1}(t)\right), \ldots, h\left(t_{0}, \varphi, y\right)\left(\tau_{s}(t)\right)\right)
$$

с начальным условием

$$
y\left(t_{0}\right)=x_{0},
$$

где оператор $h(\cdot)$ определяется по формуле

$$
h\left(t_{0}, \varphi, y\right)(t)= \begin{cases}\varphi(t), & t \in\left[\tau_{0}, t_{0}\right) \\ y(t), & t \in\left[t_{0}, b\right]\end{cases}
$$

ОПРЕДЕЛЕНИЕ 2.1. Пусть $\mu=\left(t_{0}, x_{0}, \varphi, f\right) \in A$. Абсолютно непрерывную функцию $y(t)=y(t, \mu) \in O, t \in\left[r_{1}, r_{2}\right] \subset J$, будем называть решением уравнения (2.1) с начальным условием (2.2) или решением, соответствующим әлементу $\mu$ и определенным на интервале $\left[r_{1}, r_{2}\right]$, если $t_{0} \in\left[r_{1}, r_{2}\right], y\left(t_{0}\right)=x_{0}$ и $y(t)$ на интервале $\left[r_{1}, r_{2}\right]$ п.в. удовлетворяет уравнению $(2.1)$.

ЗАмечание 2.1. Пусть $y(t, \mu), t \in\left[r_{1}, r_{2}\right], \mu \in A,-$ решение уравнения $(2.1)$ с начальным условием (2.2). Тогда, как легко видеть, функция

$$
x(t, \mu)=h\left(t_{0}, \varphi, y(\cdot, \mu)\right)(t), \quad t \in\left[\tau_{0}, r_{2}\right]
$$

будет решением уравнения (1.1) с начальньм условием (1.2) (см. определение 1.1, $(2.3))$. 
Лемма 2.1. Пусть $\widetilde{y}(t)$ - соответствующее әлементу $\widetilde{\mu} \in A$ решение, определенное на $\left[r_{1}, r_{2}\right] \subset(a, b), K_{1} \subset O$ - компакт, содержащий некоторую окрестность множества $\widetilde{\varphi}\left(J_{1}\right) \cup \widetilde{y}\left(\left[r_{1}, r_{2}\right]\right)$. Тогда существуют такие числа $\varepsilon_{1}>0, \delta_{1}>0$, что для произвольного әлемента $(\varepsilon, \delta \mu) \in\left[0, \varepsilon_{1}\right] \times V$ әлемент $\widetilde{\mu}+\varepsilon \delta \mu \in A$ и ему соответствует решение $y(t, \tilde{\mu}+\varepsilon \delta \mu)$, определенное на $\left[r_{1}-\delta_{1}, r_{2}+\delta_{1}\right] \subset J . \Pi р и$ этом

$$
\begin{gathered}
\varphi(t)=\widetilde{\varphi}(t)+\varepsilon \delta \varphi(t) \in K_{1}, \quad t \in J_{1} ; \\
y(t, \widetilde{\mu}+\varepsilon \delta \mu) \in K_{1}, \quad t \in\left[r_{1}-\delta_{1}, r_{2}+\delta_{1}\right] ; \\
\lim _{\varepsilon \rightarrow 0} y(t, \widetilde{\mu}+\varepsilon \delta \mu)=y(t, \widetilde{\mu})
\end{gathered}
$$

равномерно по $(t, \delta \mu) \in\left[r_{1}-\delta_{1}, r_{2}+\delta_{1}\right] \times V$.

Эта лемма является аналогом леммы 2.1 [5] и доказьвается аналогичньм способом.

Лемма 2.2. Пусть $\widetilde{x}(t)$ - соответствующее әлементу $\widetilde{\mu} \in A$ решение, определенное на $\left[\tau_{0}, \widetilde{t}_{1}\right], \widetilde{t}_{i} \in(a, b), i=0,1, K_{1} \subset O$ - компакт, содержащий некоторую окрестность множества $\widetilde{\varphi}\left(J_{1}\right) \cup \widetilde{x}\left(\left[\widetilde{t}_{0}, \widetilde{t}_{1}\right]\right)$. Тогда существуют такие числа $\varepsilon_{1}>0, \delta_{1}>0$, что для произвольного әлемента $(\varepsilon, \delta \mu) \in$ $\left[0, \varepsilon_{1}\right] \times V$ әлемент $\widetilde{\mu}+\varepsilon \delta \mu \in A$ и ему соответствует решение $x(t, \widetilde{\mu}+\varepsilon \delta \mu)$, определенное на $\left[\tau_{0}, \widetilde{t}_{1}+\delta_{1}\right] \subset J_{1}$. При этом

$$
x(t, \widetilde{\mu}+\varepsilon \delta \mu) \in K_{1}, \quad t \in\left[\tau_{0}, \widetilde{t}_{1}+\delta_{1}\right] .
$$

Нетрудно заметить, что если в лемме $1.1 r_{1}=\widetilde{t}_{0}, r_{2}=\widetilde{t}_{1}$, то $\widetilde{y}(t)=\widetilde{x}(t)$, $t \in\left[\widetilde{t}_{0}, \widetilde{t}_{2}\right] ; x(t, \widetilde{\mu}+\varepsilon \delta \mu)=h\left(t_{0}, \varphi, y(\cdot, \widetilde{\mu}+\varepsilon \delta \mu)\right)(t)$ при $t \in\left[\tau_{0}, \widetilde{t}_{1}+\delta_{1}\right], \varepsilon \in\left[0, \varepsilon_{1}\right]$, $\delta \mu \in V$ (см. (2.4)).

Следовательно, лемма 2.2 является непосредственным следствием леммы 2.1 (см. (2.5)).

В силу единственности решение $y(t, \widetilde{\mu})$ на отрезке $\left[r_{1}-\delta_{1}, r_{2}+\delta_{1}\right]$ является продолжением решения $\widetilde{y}(t)$. Поэтому в дальнейшем мы можем считать, что решение $\widetilde{y}(t)$ с самого начала определено на всем отрезке $\left[r_{1}-\delta_{1}, r_{2}+\delta_{1}\right]$.

По определению прирашение решения $\widetilde{y}(t)=y(t, \widetilde{\mu})$ есть

$$
\begin{gathered}
\Delta y(t)=\Delta y(t, \varepsilon \delta \mu)=y(t, \widetilde{\mu}+\varepsilon \delta \mu)-\widetilde{y}(t), \\
(t, \varepsilon, \delta \mu) \in\left[r_{1}-\delta_{1}, r_{2}+\delta_{1}\right] \times[0, \varepsilon] \times V .
\end{gathered}
$$

Очевидно (см. лемму 2.1), что

$$
\lim _{\varepsilon \rightarrow 0} \Delta y(t, \varepsilon \delta \mu)=0
$$

равномерно по $(t, \delta \mu) \in\left[r_{1}-\delta_{1}, r_{2}+\delta_{1}\right] \times V$.

Лемма 2.3 [5]. Для произвольного компакта $K \subset O$ u $f \in E$ существует такая функция $L_{f, K}(\cdot) \in L\left(J, \mathbb{R}_{+}\right)$, что почти для всех $t \in J$ и для любъх $x_{i}^{\prime}, x_{i}^{\prime \prime} \in K, i=1, \ldots, s$, выполнено неравенство

$$
\left|f\left(t, x_{1}^{\prime}, \ldots, x_{s}^{\prime}\right)-f\left(t, x_{1}^{\prime \prime}, \ldots, x_{s}^{\prime \prime}\right)\right| \leqslant L_{f, K}(t) \sum_{i=1}^{s}\left|x_{i}^{\prime}-x_{i}^{\prime \prime}\right| .
$$


Лемма 2.4. Пусть $\gamma_{i}^{0}=\widetilde{t}_{0}, i=1, \ldots, p, \gamma_{p+1}^{0}<\cdots<\gamma_{s}^{0} \leqslant r_{2}$, u пусть выполнены условия 1.2) и 1.3) теоремы 1.1. Тогда существует такое число $\varepsilon_{2}>0$, что для произвольного $(\varepsilon, \delta \mu) \in\left[0, \varepsilon_{2}\right] \times V$ выполнено неравенство

$$
\max _{t \in\left[\bar{t}_{0}, r_{2}+\delta_{1}\right]}|\Delta y(t)| \leqslant O(\varepsilon \delta \mu) .
$$

При этом

$$
\Delta y\left(\widetilde{t}_{0}\right)=\varepsilon\left(\delta x_{0}+\left(\sum_{i=0}^{p}\left(\widehat{\gamma}_{i+1}^{-}-\widehat{\gamma}_{i}^{-}\right) f_{i}^{-}\right) \delta t_{0}\right)+o(\varepsilon \delta \mu)
$$

ДокАЗАТЕльСтво. Пусть $\varepsilon_{2} \in\left(0, \varepsilon_{1}\right)$ настолько мало, что для произвольного $(\varepsilon, \delta \mu) \in\left[0, \varepsilon_{2}\right] \times V^{-}$выполнены соотношения

$$
\begin{gathered}
t_{0}=\widetilde{t}_{0}+\varepsilon \delta t_{0} \in\left(\widetilde{t}_{0}-\delta, \widetilde{t}_{0}\right] \\
\widetilde{t}_{0}<\gamma_{p+1}\left(t_{0}\right)<\gamma_{p+1}^{0}<\gamma_{p+2}\left(t_{0}\right)<\cdots<\gamma_{s-1}^{0}<\gamma_{s}\left(t_{0}\right) .
\end{gathered}
$$

Функция $\Delta y(t)$ на интервале $\left[\widetilde{t}_{0}, r_{2}+\delta_{1}\right]$ удовлетворяет уравнению

$$
\dot{\Delta} y(t)=a(t, \varepsilon \delta \mu)+b(t, \varepsilon \delta \mu),
$$

где

$$
\begin{aligned}
a(t, \varepsilon \delta \mu) & =\widetilde{f}\left(t_{0}, \varphi, \widetilde{y}+\Delta y\right)(t)-\widetilde{f}\left(\widetilde{t_{0}}, \widetilde{\varphi}, \widetilde{y}\right)(t), \\
b(t, \varepsilon \delta \mu) & =\varepsilon \delta f\left(t_{0}, \varphi, \widetilde{y}+\Delta y\right)(t) .
\end{aligned}
$$

Уравнение (2.13) перепишем в интегральной форме

$$
\Delta y(t)=\Delta y\left(\widetilde{t}_{0}\right)+\int_{\widetilde{t}_{0}}^{t}(a(\xi, \varepsilon \delta \mu)+b(\xi, \varepsilon \delta \mu)) d \xi, \quad t \in\left[\widetilde{t}_{0}, r_{2}+\delta_{1}\right] .
$$

Отсюда следует

$$
\begin{aligned}
|\Delta y(t)| & \leqslant\left|\Delta y\left(\widetilde{t_{0}}\right)\right|+\int_{\widetilde{t}_{0}}^{t}|a(\xi, \varepsilon \delta \mu)| d \xi+\int_{\widetilde{t}_{0}}^{r_{2}+\delta_{1}}|b(\xi, \varepsilon \delta \mu)| d \xi \\
& =\left|\Delta y\left(\widetilde{t_{0}}\right)\right|+a_{1}(t, \varepsilon \delta \mu)+b_{1}(\varepsilon \delta \mu) .
\end{aligned}
$$

Докажем формулу (2.11). Имеем

$$
\Delta y\left(\widetilde{t}_{0}\right)=y\left(\widetilde{t_{0}}, \widetilde{\mu}+\varepsilon \delta \mu\right)-\widetilde{y}\left(\widetilde{t}_{0}\right)=\varepsilon \delta x_{0}+\int_{t_{0}}^{\widetilde{t}_{0}}\left(\widetilde{f}\left(t_{0}, \varphi, \widetilde{y}+\Delta y\right)(t)+b(t, \varepsilon \delta \mu)\right) d t .
$$

Ho

$$
h\left(t_{0}, \varphi, \widetilde{y}+\Delta y\right)\left(\tau_{i}(t)\right) \in K_{1}, \quad t \in\left[r_{1}-\delta_{1}, r_{2}+\delta_{1}\right], \quad i=1, \ldots, s
$$

(см. $(2.5))$. 
Поэтому

$$
|b(t, \varepsilon \delta \mu)| \leqslant \varepsilon \alpha \sum_{i=1}^{k} m_{\delta f_{i}, K_{1}}(t), \quad t \in\left[r_{1}-\delta_{1}, r_{2}+\delta_{1}\right]
$$

(см. (2.14), (1.3)).

Следовательно,

$$
\Delta y\left(\widetilde{t_{0}}\right)=\varepsilon \delta x_{0}+\int_{t_{0}}^{\widetilde{t}_{0}} \widetilde{f}\left(t_{0}, \varphi, \widetilde{y}+\Delta y\right)(t) d t+o(\varepsilon \delta \mu) .
$$

Теперь преобразуем интегральное слагаемое

$$
\begin{gathered}
\int_{t_{0}}^{\widetilde{t}_{0}} \tilde{f}\left(t_{0}, \varphi, \widetilde{y}+\Delta y\right)(t) d t=\sum_{i=0}^{p} \int_{\varrho_{i}\left(t_{0}\right)}^{\varrho_{i+1}\left(t_{0}\right)} \tilde{f}\left(t, \widetilde{y}\left(\tau_{1}(t)\right)+\Delta y\left(\tau_{1}(t)\right), \ldots, \widetilde{y}\left(\tau_{i}(t)\right)\right. \\
\left.+\Delta y\left(\tau_{i}(t)\right), \varphi\left(\tau_{i+1}(t)\right), \ldots, \varphi\left(\tau_{s}(t)\right)\right) d t=\sum_{i=0}^{p} I_{i} \\
\varrho_{0}(t)=t, \quad \varrho_{p+1}\left(t_{0}\right)=\widetilde{t}_{0}, \quad \varrho_{i}(t)=\gamma_{i}(t), \quad i=1, \ldots, p
\end{gathered}
$$

(см. условие 1.2), (2.3)).

Ясно, что

$$
\begin{aligned}
I_{0} & =\varepsilon\left(\dot{\gamma}_{1}^{-}-1\right) f_{0}^{-} \delta t_{0}+o(\varepsilon \delta \mu)+\int_{t_{0}}^{\gamma_{1}\left(t_{0}\right)}\left(\tilde{f}\left(t, \varphi\left(\tau_{1}(t)\right), \ldots, \varphi\left(\tau_{s}(t)\right)\right)-f_{0}^{-}\right) d t \\
& =\varepsilon\left(\dot{\gamma}_{1}^{-}-1\right) f_{0}^{-} \delta t_{0}+o(\varepsilon \delta \mu)+\alpha(\varepsilon \delta \mu) .
\end{aligned}
$$

Покажем, что

$$
\alpha(\varepsilon \delta \mu)=o(\varepsilon \delta \mu) .
$$

В самом деле, согласно условию 1.3) будем иметь

$$
\begin{gathered}
\lim _{\varepsilon \rightarrow 0} \sup _{t \in\left[t_{0}, \gamma_{1}\left(t_{0}\right)\right]}\left|\tilde{f}\left(t, \varphi\left(\tau_{1}(t)\right), \ldots, \varphi\left(\tau_{s}(t)\right)\right)-f_{0}^{-}\right|=\lim _{\omega \rightarrow \sigma_{0}}\left|\tilde{f}(\omega)-f_{0}^{-}\right|=0, \\
\omega \in\left(\widetilde{t_{0}}-\delta, \widetilde{t_{0}}\right] \times O^{s} .
\end{gathered}
$$

Отсюда непосредственно вытекает (2.20). Аналогичным образом доказываются равенства

$$
I_{i}=\varepsilon\left(\dot{\gamma}_{i+1}^{-}-\dot{\gamma}_{i}^{-}\right) f_{i}^{-} \delta t_{0}+o(\varepsilon \delta \mu), \quad i=1, \ldots, p-1, \quad I_{p}=-\varepsilon \dot{\gamma}_{p}^{-} \delta t_{0}+o(\varepsilon \delta \mu) .
$$

Из (2.17) с учетом (2.18)-(2.21) следует (2.11). Прежде чем доказать неравенство (2.10), заметим, что если $i=p+1, \ldots, s, \xi \in\left[\gamma_{i}\left(t_{0}\right), \gamma_{i}^{0}\right]$, то

$$
\begin{aligned}
& \lim _{\varepsilon \rightarrow 0}\left(\xi, \widetilde{y}\left(\tau_{1}(\xi)\right)+\Delta y\left(\tau_{1}(\xi)\right), \ldots, \widetilde{y}\left(\tau_{i}(\xi)\right)\right. \\
&\left.+\Delta y\left(\tau_{i}(\xi)\right), \varphi\left(\tau_{i+1}(\xi)\right), \ldots, \varphi\left(\tau_{s}(\xi)\right)\right)=\sigma_{i} \\
& \lim _{\varepsilon \rightarrow 0}\left(\xi, \widetilde{y}\left(\tau_{1}(\xi)\right), \ldots, \widetilde{y}\left(\tau_{i-1}(\xi)\right), \widetilde{\varphi}\left(\tau_{i}(\xi)\right), \ldots, \widetilde{\varphi}\left(\tau_{s}(\xi)\right)\right)=\sigma_{i}^{0}
\end{aligned}
$$

(cM. (2.8)). 
Таким образом, в силу условия 1.3$)$ для достаточно малого $\varepsilon_{2} \in\left(0, \varepsilon_{1}\right]$ функции

$$
\sup _{t \in\left[t_{0}, \widetilde{t}_{0}\right]} \dot{\gamma}_{i}(t), \sup _{t \in\left[\gamma_{i}\left(t_{0}\right), \gamma_{i}^{0}\right]}|a(t, \varepsilon \delta \mu)|, \quad i=p+1, \ldots, s,
$$

ограничены на множестве $\left[0, \varepsilon_{2}\right] \times V^{-}$.

Следовательно, для произвольного $(\varepsilon, \delta \mu) \in\left[0, \varepsilon_{2}\right] \times V^{-}$справедливы оценки

$$
\int_{\gamma_{i}\left(t_{0}\right)}^{\gamma_{i}^{0}}|a(t, \varepsilon \delta \mu)| d t \leqslant O(\varepsilon \delta \mu), \quad i=p+1, \ldots, s .
$$

Теперь оценим $a_{1}(t, \varepsilon \delta \mu), t \in\left[\widetilde{t_{0}}, r_{2}+\delta_{1}\right]$. Для этого рассмотрим несколько случаев.

Пусть $t \in\left[\widetilde{t}_{0}, \gamma_{p+1}\left(t_{0}\right)\right]$. Тогда, учитывая вид оператора $h(\cdot)$ и неравенство (2.9), будем иметь (см. (2.5))

$$
\begin{aligned}
a_{1}(t, \varepsilon \delta \mu) & \leqslant \int_{\widetilde{t}_{0}}^{t} L_{\widetilde{f}, K_{1}}(t)\left(\sum_{i=1}^{p}\left|\Delta y\left(\tau_{i}(\xi)\right)\right|+\varepsilon \sum_{i=p+1}^{s}\left|\delta \varphi\left(\tau_{i}(t)\right)\right|\right) d \xi \\
& \leqslant O(\varepsilon \delta \mu)+\sum_{i=1}^{p} \int_{\tau_{i}\left(\widetilde{t}_{0}\right)}^{\tau_{i}(t)} L_{\widetilde{f}, K_{1}}\left(\gamma_{i}(\xi)\right) \dot{\gamma}_{i}(\xi)|\Delta y(\xi)| d \xi \\
& \leqslant O(\varepsilon \delta \mu)+\int_{\widetilde{t}_{0}}^{t} L(\xi)|\Delta y(\xi)| d \xi
\end{aligned}
$$

где

$$
L(\xi)=\sum_{i=1}^{s} \chi\left(\gamma_{i}(\xi)\right) L_{\widetilde{f}, K_{1}}\left(\gamma_{i}(\xi)\right) \dot{\gamma}_{i}(\xi),
$$

$\chi(\xi)$ - характеристическая функция интервала $J$. При $\gamma_{i}(\xi)>b$ предполагается, что

$$
\chi\left(\gamma_{i}(\xi)\right) L_{\widetilde{f}, K_{1}}\left(\gamma_{i}(\xi)\right)=0 .
$$

Если $t \in\left[\gamma_{p+1}\left(t_{0}\right), \gamma_{p+1}^{0}\right]$, то согласно $(2.22),(2.23)$ получим

$$
\begin{aligned}
a_{1}(t, \varepsilon \delta \mu) & \leqslant a_{1}\left(\gamma_{p+1}\left(t_{0}\right), \varepsilon \delta \mu\right)+\int_{\gamma_{p+1}\left(t_{0}\right)}^{\gamma_{p+1}^{0}}|a(\xi, \varepsilon \delta \mu)| d \xi \\
& \leqslant O(\varepsilon \delta \mu)+\int_{\widetilde{t}_{0}}^{t} L(\xi) \mid \Delta y(\xi) d \xi .
\end{aligned}
$$

Таким образом, оценка (2.23) справедлива на всем интервале $\left[\widetilde{t}_{0}, \gamma_{p+1}^{0}\right]$.

Пусть $t \in\left[\gamma_{p+1}^{0}, \gamma_{p+2}\left(t_{0}\right)\right]$, тогда

$$
\begin{aligned}
& a_{1}(t, \varepsilon \delta \mu) \\
& \quad \leqslant a_{1}\left(\gamma_{p+1}^{0}, \varepsilon \delta \mu\right)+\int_{\gamma_{p+1}^{0}}^{t} L_{\widetilde{f}, K_{1}}(\xi)\left(\sum_{i=1}^{p+1}\left|\Delta y\left(\tau_{i}(\xi)\right)\right|+\varepsilon \sum_{i=p+2}^{s}\left|\delta \varphi\left(\tau_{i}(\xi)\right)\right|\right) d \xi \\
& \quad \leqslant a_{1}\left(\gamma_{p+1}^{0}, \varepsilon \delta \mu\right)+\sum_{i=1}^{p+1} \int_{\tau_{i}\left(\gamma_{p+1}\right)}^{\tau_{i}(t)} L_{\widetilde{f}, K_{1}}\left(\gamma_{i}(\xi)\right) \dot{\gamma}_{i}(\xi)|\Delta y(\xi)| d \xi+O(\varepsilon \delta \mu) .
\end{aligned}
$$


Так как $\tau_{i}\left(\gamma_{p+1}^{0}\right) \geqslant \widetilde{t}_{0}, \tau_{i}(t) \leqslant t, i=1, \ldots, p+1$, то полученное неравенство можно записать следующим образом:

$$
a_{1}(t, \varepsilon \delta \mu) \leqslant O(\varepsilon \delta \mu)+a_{1}\left(\gamma_{p+1}^{0}, \varepsilon \delta \mu\right)+\int_{\tilde{t}_{0}}^{t} L(\xi)|\Delta y(\xi)| d \xi
$$

Итак, при $t \in\left[\widetilde{t_{0}}, \gamma_{p+2}\left(t_{0}\right)\right]$ справедлива оценка

$$
a_{1}(t, \varepsilon \delta \mu) \leqslant O(\varepsilon \delta \mu)+2 \int_{\bar{t}_{0}}^{t} L(\xi)|\Delta y(\xi)| d \xi .
$$

Аналогичным рассуждением, используя (2.22), можно установить справедливость неравенства $(2.25)$ и на интервале $\left[\widetilde{t}_{0}, \gamma_{p+2}^{0}\right]$.

Продолжая этот процесс, докажем, что

$$
\begin{gathered}
a_{1}(t, \varepsilon \delta \mu) \leqslant O(\varepsilon \delta \mu)+(i+1) \int_{\widetilde{t}_{0}}^{t} L(\xi)|\Delta y(\xi)| d \xi, \\
t \in\left[\widetilde{t_{0}}, \gamma_{p+i+1}^{0}\right], \quad i=1, \ldots, s-p-1 .
\end{gathered}
$$

Далее, если $t \in\left[\gamma_{s}^{0}, r_{2}+\delta_{1}\right]$, то

$$
\begin{aligned}
a_{1}(t, \varepsilon \delta \mu) & \leqslant a_{1}\left(\gamma_{s}^{0}, \varepsilon \delta \mu\right)+\sum_{i=1}^{s} \int_{\gamma_{s}^{0}}^{t} L_{\widetilde{f}, K_{1}}(\xi)\left|\Delta y\left(\tau_{i}(\xi)\right)\right| d \xi \\
& =a_{1}\left(\gamma_{s}^{0}, \varepsilon \delta \mu\right)+\sum_{i=1}^{s} \int_{\tau_{i}\left(\gamma_{i}^{0}\right)}^{\tau_{i}(t)} L_{\widetilde{f}, K_{1}}\left(\gamma_{i}(\xi)\right) \dot{\gamma}_{i}(\xi)|\Delta y(\xi)| d \xi
\end{aligned}
$$

В силу неравенств $\tau_{i}\left(\gamma_{s}^{0}\right) \geqslant \widetilde{t}_{0}, i=1, \ldots, s$, имеем

$$
\begin{aligned}
a_{1}(t, \varepsilon \delta \mu) & \leqslant a_{1}\left(\gamma_{s}^{0}, \varepsilon \delta \mu\right)+\int_{\tilde{t}_{0}}^{t} L(\xi)|\Delta y(\xi)| d \xi \\
& \leqslant O(\varepsilon \delta \mu)+(s-p+1) \int_{\widetilde{t}_{0}}^{t} L(\xi)|\Delta y(\xi)| d \xi, \quad t \in\left[\gamma_{s}^{0}, r_{2}+\delta_{1}\right] .
\end{aligned}
$$

Таким образом, на отрезке $\left[\widetilde{t}_{0}, r_{2}+\delta_{1}\right]$ вьполнено

$$
a_{1}(t, \varepsilon \delta \mu) \leqslant O(\varepsilon \delta \mu)+(s-p+1) \int_{\widetilde{t}_{0}}^{t} L(\xi)|\Delta y(\xi)| d \xi .
$$

В силу (2.16)

$$
b_{1}(\varepsilon \delta \mu)=O(\varepsilon \delta \mu)
$$

Согласно $(2.11),(2.26),(2.27)$ из неравенства (2.15) непосредственно следует

$$
|\Delta y(t)| \leqslant O(\varepsilon \delta \mu)+(s-p+1) \int_{\widetilde{t}_{0}}^{t} L(\xi)|\Delta y(\xi)| d \xi, \quad t \in\left[\widetilde{t}_{0}, r_{2}+\delta_{1}\right] .
$$

Из этого в силу леммы Гронуолла вытекает (2.10). 
ЛЕмма 2.5. Пусть $\gamma_{i}^{0}=\widetilde{t}_{0}, i=1, \ldots, p, \gamma_{p+1}^{0}<\cdots<\gamma_{s}^{0} \leqslant r_{2}$, u nусть выполнены условия 1.4) и 1.5) теоремы 1.2. Тогда существует такое число $\varepsilon_{2}>0$, что для произвольного әлемента $(\varepsilon, \delta \mu) \in\left[0, \varepsilon_{2}\right] \times V^{+}$выполнено неравенство

$$
\max _{t \in\left[t_{0}, r_{2}+\delta_{1}\right]}|\Delta y(t)| \leqslant O(\varepsilon \delta \mu)
$$

При этом

$$
\Delta y\left(t_{0}\right)=\varepsilon\left(\delta x_{0}-f_{p}^{+} \delta t_{0}\right)+o(\varepsilon \delta \mu)
$$

ДокАЗАТЕЛЬСтво. Пусть $\varepsilon_{2} \in\left(0, \varepsilon_{1}\right]$ настолько мало, что для произвольного элемента $(\varepsilon, \delta \mu) \in\left[0, \varepsilon_{2}\right] \times V^{+}$выполнены соотношения

$$
\begin{gathered}
t_{0} \in\left[\widetilde{t}_{0}, \widetilde{t}_{0}+\delta\right] \\
\gamma_{p}\left(t_{0}\right)<\gamma_{p+1}^{0}<\gamma_{p+1}\left(t_{0}\right)<\gamma_{p+2}^{0}<\cdots<\gamma_{s}^{0}<\gamma_{s}\left(t_{0}\right)<r_{2}+\delta_{1} .
\end{gathered}
$$

Функция $\Delta y(t)$ на интервале $\left[t_{0}, r_{2}+\delta_{1}\right]$ удовлетворяет уравнению $(2.13)$, которое запишем в интегральной форме

$$
\Delta y(t)=\Delta y\left(t_{0}\right)+\int_{t_{0}}^{t}(a(\xi, \varepsilon \delta \mu)+b(\xi, \varepsilon \delta \mu)) d \xi, \quad t \in\left[t_{0}, r_{2}+\delta_{1}\right]
$$

Откуда следует

$$
\begin{aligned}
|\Delta y(t)| & \leqslant\left|\Delta y\left(t_{0}\right)\right|+\int_{t_{0}}^{t}|a(\xi, \varepsilon \delta \mu)| d \xi+\int_{t_{0}}^{r_{2}+\delta_{1}}|b(\xi, \varepsilon \delta \mu)| d \xi \\
& =\left|\Delta y\left(t_{0}\right)\right|+a_{2}(t, \varepsilon \delta \mu)+b_{2}(\varepsilon \delta \mu) .
\end{aligned}
$$

Докажем формулу (2.29). Имеем

$$
\begin{aligned}
\Delta y\left(t_{0}\right) & =\varepsilon \delta x_{0}-\int_{\widetilde{t}_{0}}^{t_{0}} \widetilde{f}\left(\widetilde{t_{0}}, \widetilde{\varphi}, \widetilde{y}\right)(t) d t \\
& =\varepsilon \delta x_{0}-\int_{\tilde{t}_{0}}^{t_{0}} \widetilde{f}\left(t, \widetilde{y}\left(\tau_{1}(t)\right), \ldots, \widetilde{y}\left(\tau_{p}(t)\right), \widetilde{\varphi}\left(\tau_{p+1}(t)\right), \ldots, \widetilde{\varphi}\left(\tau_{s}(t)\right)\right) d t \\
& =\varepsilon\left(\delta x_{0}-f_{p}^{+} \delta t_{0}\right)+o(\varepsilon \delta \mu) .
\end{aligned}
$$

В силу условия 1.5$)$ для достаточно малого $\varepsilon_{2} \in\left(0, \varepsilon_{1}\right]$ функции

$$
\begin{array}{ll}
\sup _{t \in\left[\bar{t}_{0}, t_{0}\right]} \dot{\gamma}_{i}(t), & i=1, \ldots, s, \\
\sup _{t \in\left[\gamma_{i-1}\left(t_{0}\right), \gamma_{i}\left(t_{0}\right)\right]}|a(t, \varepsilon \delta \mu)|, & i=1, \ldots, p, \quad \gamma_{0}\left(t_{0}\right)=t_{0}, \\
\sup _{t \in\left[\gamma_{i}^{0}, \gamma_{i}\left(t_{0}\right)\right]}|a(t, \varepsilon \delta \mu)|, & i=p+1, \ldots, s,
\end{array}
$$


ограничены на множестве $\left[0, \varepsilon_{2}\right] \times V^{+}$. Ясно, что при $i=1, \ldots, p$

$$
\left|\gamma_{i}\left(t_{0}\right)-\gamma_{i-1}\left(t_{0}\right)\right| \leqslant\left|\gamma_{i}\left(t_{0}\right)-\gamma_{i}\left(\widetilde{t}_{0}\right)\right|+\left|\gamma_{i-1}\left(\widetilde{t}_{0}\right)-\gamma_{i-1}\left(t_{0}\right)\right| \leqslant O(\varepsilon \delta \mu) .
$$

Из этих условий вытекает, что для произвольного элемента $(\varepsilon, \delta \mu) \in\left[0, \varepsilon_{2}\right] \times V^{+}$ справедливы оценки

$$
\begin{gathered}
\int_{\gamma_{i-1}\left(t_{0}\right)}^{\gamma_{i}\left(t_{0}\right)}|a(\xi, \varepsilon \delta \mu)| d \xi \leqslant O(\varepsilon \delta \mu), \quad i=1, \ldots, p, \\
\int_{\gamma_{i}^{0}}^{\gamma_{i}\left(t_{0}\right)}|a(\xi, \varepsilon \delta \mu)| d \xi \leqslant O(\varepsilon \delta \mu), \quad i=p+1, \ldots, s .
\end{gathered}
$$

Теперь оценим $a_{2}(t, \varepsilon \delta \mu), t \in\left[t_{0}, r_{2}+\delta_{1}\right]$. Для этого рассмотрим несколько случаев.

Пусть $t \in\left[t_{0}, \gamma_{p}\left(t_{0}\right)\right]$. Тогда

$$
a_{2}(t, \varepsilon \delta \mu) \leqslant \sum_{i=1}^{p} \int_{\gamma_{i-1}\left(t_{0}\right)}^{\gamma_{i}\left(t_{0}\right)}|a(\xi, \varepsilon \delta \mu)| d \xi \leqslant O(\varepsilon \delta \mu)
$$

(см. (2.32)).

Если $t \in\left[\gamma_{p}\left(t_{0}\right), \gamma_{p+1}^{0}\right]$, то

$$
\begin{aligned}
a_{2}(t, \varepsilon \delta \mu) \leqslant & a_{2}\left(\gamma_{p}\left(t_{0}\right), \varepsilon \delta \mu\right) \\
& +\int_{\gamma_{p}\left(t_{0}\right)}^{t} L_{\widetilde{f}, K_{1}}(\xi)\left(\sum_{i=1}^{p}\left|\Delta y\left(\tau_{i}(\xi)\right)\right|+\varepsilon \sum_{i=p+1}^{s}\left|\delta \varphi\left(\tau_{i}(\xi)\right)\right|\right) d \xi \\
\leqslant & O(\varepsilon \delta \mu)+\sum_{i=0}^{p} \int_{\tau_{i}\left(\gamma_{p}\left(t_{0}\right)\right)}^{\tau_{i}(t)} L_{\widetilde{f}, K_{1}}\left(\gamma_{i}(\xi)\right) \dot{\gamma}_{i}(\xi)|\Delta y(\xi)| d \xi
\end{aligned}
$$

Так как

$$
\tau_{i}\left(\gamma_{p}\left(t_{0}\right)\right) \geqslant \tau_{i}\left(\gamma_{i}\left(t_{0}\right)\right)=t_{0}, \quad \tau_{i}(t) \leqslant t, \quad i=1, \ldots, p,
$$

то

$$
a_{2}(t, \varepsilon \delta \mu) \leqslant O(\varepsilon \delta \mu)+\int_{t_{0}}^{t} L(\xi)|\Delta y(\xi)| d \xi, \quad t \in\left[t_{0}, \gamma_{p+1}^{0}\right]
$$

(см. $(2.24),(2.33))$.

После этого с учетом (2.29), (2.32) аналогичным способом (см. доказательство леммы 2.4) получаем

$$
|\Delta y(t)| \leqslant O(\varepsilon \delta \mu)+(s-p+1) \int_{t_{0}}^{t} L(\xi)|\Delta y(\xi)| d \xi, \quad t \in\left[t_{0}, r_{2}+\delta_{1}\right]
$$

(см. (2.31)).

Из этого в силу леммы Гронуолла следует (2.28). 


\section{§3. Доказательство теоремы 1.1}

Пусть $r_{1}=\widetilde{t}_{0}, r_{2}=\widetilde{t}_{1}$, тогда для произвольного элемента $(\varepsilon, \delta \mu) \in\left[0, \varepsilon_{1}\right] \times$ $V^{-}$решение $y(t, \widetilde{\mu}+\varepsilon \delta \mu)$ определено на $\left[\widetilde{t}_{0}-\delta_{1}, \widetilde{t}_{1}+\delta_{1}\right]$, а решение $x(t, \widetilde{\mu}+\varepsilon \delta \mu)$ определено на $\left[\tau_{0}, \widetilde{t}_{1}+\delta_{1}\right]$, при этом

$$
y(t, \tilde{\mu}+\varepsilon \delta \mu)=x(t, \tilde{\mu}+\varepsilon \delta \mu), \quad t \in\left[t_{0}, \widetilde{t}_{1}+\delta_{1}\right]
$$

(см. леммы 2.1, 2.2, (2.4)). Таким образом, (см. (1.4), (2.7))

$$
\Delta x(t)= \begin{cases}\varepsilon \delta \varphi(t), & t \in\left[\tau_{0}, t_{0}\right) ; \\ y(t, \widetilde{\mu}+\varepsilon \delta \mu)-\widetilde{\varphi}(t), & t \in\left[t_{0}, \widetilde{t}_{0}\right) ; \\ \Delta y(t), & t \in\left[\widetilde{t_{0}}, \widetilde{t}_{1}+\delta_{1}\right] .\end{cases}
$$

Пусть $\delta_{2} \in\left(0, \min \left\{\delta_{1}, \widetilde{t}_{1}-\gamma_{s}\right\}\right)$. В силу леммы 2.4 сушествует такое число $\varepsilon_{2} \in\left(0, \varepsilon_{1}\right]$, что

$$
\begin{gathered}
|\Delta x(t)| \leqslant O(\varepsilon \delta \mu) \quad \forall(t, \varepsilon, \delta \mu) \in\left[\widetilde{t}_{0}, \widetilde{t}_{1}+\delta_{2}\right] \times\left[0, \varepsilon_{2}\right] \times V^{-}, \\
\Delta x\left(\widetilde{t}_{0}\right)=\varepsilon\left(\delta x_{0}+\left(\sum_{i=0}^{p}\left(\widehat{\gamma}_{i+1}^{-}-\widehat{\gamma}_{i}^{-}\right) f_{i}^{-}\right) \delta t_{0}\right)+o(\varepsilon \delta \mu)
\end{gathered}
$$

(см. (3.1)).

Функция $\Delta x(t)$ на интервале $\left[\widetilde{t}_{0}, \widetilde{t}_{1}+\delta_{2}\right]$ удовлетворяет уравнению

$$
\dot{\Delta} x(t)=\sum_{i=1}^{s} \widetilde{f}_{x_{i}}[t] \Delta x\left(\tau_{i}(t)\right)+\varepsilon \delta f[t]+\sum_{i=1}^{2} R_{i}(t, \varepsilon \delta \mu)
$$

где

$$
\begin{aligned}
R_{1}(t, \varepsilon \delta \mu)= & \widetilde{f}\left(t, \widetilde{x}\left(\tau_{1}(t)\right)+\Delta x\left(\tau_{1}(t)\right), \ldots, \widetilde{x}\left(\tau_{s}(t)\right)+\Delta x\left(\tau_{s}(t)\right)\right) \\
& -\widetilde{f}[t]-\sum_{i=1}^{s} \widetilde{f}_{x_{i}}[t] \Delta x\left(\tau_{i}(t)\right), \\
R_{2}(t, \varepsilon \delta \mu)= & \varepsilon\left(\delta f\left(t, \widetilde{x}\left(\tau_{1}(t)\right)+\Delta x\left(\tau_{1}(t)\right), \ldots, \widetilde{x}\left(\tau_{s}(t)\right)+\Delta x\left(\tau_{s}(t)\right)\right)-\delta f[t]\right) .
\end{aligned}
$$

Дифференциальное уравнение (3.4) будем рассматривать как линейное неоднородное уравнение с запаздываюшим аргументом. Тогда $\Delta x(t), t \in\left[\widetilde{t_{0}}, \widetilde{t}_{1}+\delta_{2}\right]$, с помошью формулы Коши может быть представлено в виде

$$
\begin{gathered}
\Delta x(t)=Y\left(\widetilde{t}_{0}, t\right) \Delta x\left(\widetilde{t}_{0}\right)+\varepsilon \int_{\widetilde{t}_{0}}^{t} Y(\xi, t) \delta f[\xi] d \xi+\sum_{i=0}^{2} h_{i}\left(t, \widetilde{t}_{0}, \varepsilon \delta \mu\right), \\
t \in\left[\widetilde{t_{0}}, \widetilde{t}_{1}+\delta_{2}\right]
\end{gathered}
$$




$$
\begin{aligned}
& \text { где } \\
& \begin{cases}h_{0}\left(t, \widetilde{t}_{0}, \varepsilon \delta \mu\right)=\sum_{i=p+1}^{s} \int_{\tau_{i}\left(\widetilde{t}_{0}\right)}^{\widetilde{t}_{0}} Y\left(\gamma_{i}(\xi), t\right) \widetilde{f}_{x_{i}}\left[\gamma_{i}(\xi)\right] \dot{\gamma}_{i}(\xi) \Delta x(\xi) d \xi, & \\
h_{i}\left(t, \widetilde{t}_{0}, \varepsilon \delta \mu\right)=\int_{\widetilde{t}_{0}}^{t} Y(\xi, t) R_{i}(\xi, \varepsilon \delta \mu) d \xi, & i=1,2,\end{cases}
\end{aligned}
$$

$Y(\xi, t)$ - матричная функция, удовлетворяющая уравнению (1.7) и условию (1.8).

В силу леммы 3.4 [5] функция $Y(\xi, t)$ непрерьвна на множестве

$$
\Pi=\{(\xi, t): a \leqslant \xi \leqslant t, t \in J\} .
$$

Поэтому

$$
Y\left(\widetilde{t}_{0}, t\right) \Delta x\left(\widetilde{t}_{0}\right)=\varepsilon Y\left(\widetilde{t}_{0}, t\right)\left(\delta x_{0}+\left(\sum_{i=0}^{p}\left(\widehat{\gamma}_{i+1}^{-}-\widehat{\gamma}_{i}^{-}\right) f_{i}^{-}\right) \delta t_{0}\right)+o(t, \varepsilon \delta \mu)
$$

(см. (3.3)).

Теперь преобразуем $h_{0}\left(t, \widetilde{t_{0}}, \varepsilon \delta \mu\right)$. Имеем

$$
\begin{aligned}
h_{0}\left(t, \widetilde{t}_{0}, \varepsilon \delta \mu\right)= & \sum_{i=p+1}^{s}\left(\varepsilon \int_{\tau_{i}\left(\widetilde{t}_{0}\right)}^{t_{0}} Y\left(\dot{\gamma}_{i}(\xi), t\right) \widetilde{f}_{x_{i}}\left[\gamma_{i}(\xi)\right] \dot{\gamma}_{i}(\xi) \delta \varphi(\xi) d \xi\right. \\
& \left.+\int_{t_{0}}^{t_{0}} Y\left(\gamma_{i}(\xi), t\right) \widetilde{f}_{x_{i}}\left[\gamma_{i}(\xi)\right] \dot{\gamma}_{i}(\xi) \Delta x(\xi) d \xi\right) \\
= & \varepsilon \sum_{i=p+1}^{s} \int_{\tau_{i}\left(\widetilde{t}_{0}\right)}^{\widetilde{t}_{0}} Y\left(\gamma_{i}(\xi), t\right) \widetilde{f}_{x_{i}}\left[\gamma_{i}(\xi)\right] \dot{\gamma}_{i}(\xi) \delta \varphi(\xi) d \xi \\
& +\sum_{i=p+1}^{s} \int_{\gamma_{i}\left(t_{0}\right)}^{\gamma_{i}^{0}} Y(\xi, t) \widetilde{f}_{x_{i}}[\xi] \Delta x\left(\tau_{i}(\xi)\right) d \xi+o(t, \varepsilon \delta \mu)
\end{aligned}
$$

(см. (2.12)).

Согласно неравенствам (2.12) выражение $h_{1}\left(t, \widetilde{t_{0}}, \varepsilon \delta \mu\right)$ при $t \in\left[\widetilde{t}_{1}-\delta_{2}, \widetilde{t}_{1}+\delta_{1}\right]$ можно представить в виде

где

$$
h_{1}\left(t, \widetilde{t_{0}}, \varepsilon \delta \mu\right)=\sum_{k=1}^{4} \alpha_{k}(t, \varepsilon \delta \mu),
$$

$$
\begin{aligned}
\alpha_{1}(t, \varepsilon \delta \mu) & =\int_{\tilde{t}_{0}}^{\gamma_{p+1}\left(t_{0}\right)} \omega(\xi, t, \varepsilon \delta \mu) d \xi \\
\alpha_{2}(t, \varepsilon \delta \mu) & =\sum_{i=p+1}^{s} \int_{\gamma_{i}\left(t_{0}\right)}^{\gamma_{i}^{0}} \omega(\xi, t, \varepsilon \delta \mu) d \xi \\
\alpha_{3}(t, \varepsilon \delta \mu) & =\sum_{i=p+1}^{s-1} \int_{\gamma_{i}^{0}}^{\gamma_{i+1}\left(t_{0}\right)} \omega(\xi, t, \varepsilon \delta \mu) d \xi \\
\alpha_{4}(t, \varepsilon \delta \mu) & =\int_{\gamma_{s}^{0}}^{t} \omega(\xi, t, \varepsilon \delta \mu) d \xi \\
\omega(\xi, t, \varepsilon \delta \mu) & =Y(\xi, t) R_{1}(\xi, \varepsilon \delta \mu) .
\end{aligned}
$$


Оценим $\alpha_{1}(t, \varepsilon \delta \mu)$ (см. (3.5)). Имеем

$$
\begin{aligned}
\mid \alpha_{1}(t, \varepsilon & \delta \mu)\left|\leqslant\|Y\| \int_{\widetilde{t}_{0}}^{\gamma_{p+1}\left(t_{0}\right)}\right| \widetilde{f}\left(t, \widetilde{x}\left(\tau_{1}(t)\right)\right. \\
& \left.+\Delta x\left(\tau_{1}(t)\right), \ldots, \widetilde{x}\left(\tau_{p}(t)\right)+\Delta x\left(\tau_{p}(t)\right), \varphi\left(\tau_{p+1}(t)\right), \ldots, \varphi\left(\tau_{s}(t)\right)\right) \\
& -\widetilde{f}\left(t, \widetilde{x}\left(\tau_{1}(t)\right), \ldots, \widetilde{x}\left(\tau_{p}(t)\right), \widetilde{\varphi}\left(\tau_{p+1}(t)\right), \ldots, \widetilde{\varphi}\left(\tau_{s}(t)\right)\right) \\
& -\sum_{i=1}^{p} \widetilde{f}_{x_{i}}[t] \Delta x\left(\tau_{i}(t)\right)-\varepsilon \sum_{i=p+1}^{s} \widetilde{f}_{x_{i}}[t] \delta \varphi\left(\tau_{i}(t)\right) \mid d t \\
\leqslant & \|Y\| \int_{\widetilde{t}_{0}}^{\widetilde{t}_{1}+\delta_{2}}\left(\int_{0}^{1} \mid \frac{d}{d \xi} \widetilde{f}\left(t, \widetilde{x}\left(\tau_{1}(t)\right)+\xi \Delta x\left(\tau_{1}(\xi)\right), \ldots, \widetilde{x}\left(\tau_{p}(\xi)\right)\right.\right. \\
& \left.+\xi \Delta x\left(\tau_{p}(t)\right), \widetilde{\varphi}\left(\tau_{p+1}(t)\right)+\xi \varepsilon \delta \varphi\left(\tau_{p+1}(t)\right), \ldots, \widetilde{\varphi}\left(\tau_{s}(t)\right)+\xi \varepsilon \delta \varphi\left(\tau_{s}(t)\right)\right) \\
& \left.-\sum_{i=1}^{p} \widetilde{f}_{x_{i}}[t] \Delta x\left(\tau_{i}(t)\right)-\varepsilon \sum_{i=p+1}^{s} \widetilde{f}_{x_{i}}[t] \delta \varphi\left(\tau_{i}(t)\right) \mid d \xi\right) d t \\
\leqslant & \|Y\| \int_{\widetilde{t}_{0}}^{\widetilde{t}_{1}+\delta_{2}}\left(\int _ { 0 } ^ { 1 } \left(\sum_{i=1}^{p} \mid \widetilde{f}_{x_{i}}\left(t, \widetilde{x}\left(\tau_{1}(t)\right)+\xi \Delta x\left(\tau_{1}(t)\right), \ldots\right)\right.\right. \\
& \left.\left.-\widetilde{f}_{x_{i}}[t]|| \delta \varphi\left(\tau_{i}(t)\right) \mid\right) d \xi\right) d t \\
\leqslant & \|Y\|\left(O(\varepsilon \delta \mu) \sum_{i=1}^{p} \sigma_{i}(\varepsilon \delta \mu)+\varepsilon \alpha \sum_{i=p+1}^{s} \sigma_{i}(\varepsilon \delta \mu)\right)
\end{aligned}
$$

где

$$
\begin{gathered}
\|Y\|=\sup _{(\xi, t) \in \Pi}|Y(\xi, t)| \\
\sigma_{i}(\varepsilon \delta \mu)=\int_{\tilde{t}_{0}}^{\widetilde{t}_{1}+\delta_{2}}\left(\int_{0}^{1}\left|\widetilde{f}_{x_{i}}\left(t, \widetilde{x}\left(\tau_{1}(t)\right)+\xi \Delta x\left(\tau_{1}(t)\right), \ldots\right)-\widetilde{f}_{x_{i}}[t]\right| d \xi\right) d t \\
i=1, \ldots, s .
\end{gathered}
$$

При $\varepsilon \rightarrow 0$

$$
\varphi(t)=\widetilde{\varphi}(t)+\varepsilon \xi \delta \varphi(t) \rightarrow \widetilde{\varphi}(t), \quad \Delta x\left(\tau_{i}(t)\right) \rightarrow 0, \quad i=1, \ldots, p,
$$

равномерно по $(\xi, t, \delta \mu) \in[0,1] \times\left[\widetilde{t}_{0}, \widetilde{t}_{1}+\delta_{2}\right] \times V^{-}$.

Поэтому в силу теоремы Лебега о предельном переходе под знаком интеграла имеем

$$
\lim _{\varepsilon \rightarrow 0} \sigma_{i}(\varepsilon \delta \mu)=0
$$

равномерно по $\delta \mu \in V^{-}$.

Таким образом,

$$
\alpha_{1}(t, \varepsilon \delta \mu)=o(t, \varepsilon \delta \mu)
$$


Теперь преобразуем $\alpha_{2}(t, \varepsilon \delta \mu)$. Нетрудно заметить, что при $t \in\left[\gamma_{i}\left(t_{0}\right), \gamma_{i}^{0}\right]$

$$
\begin{array}{ll}
\left|\Delta x\left(\tau_{j}(t)\right)\right| \leqslant O(\varepsilon \delta \mu), & j=1, \ldots, i-1, \\
\Delta x\left(\tau_{j}(t)\right)=\varepsilon \delta \varphi\left(\tau_{j}(t)\right), & j=i+1, \ldots, s, \quad i=p+1, \ldots, s
\end{array}
$$

(см. (3.1), (3.2)).

Поэтому

$$
\begin{aligned}
\int_{\gamma_{i}\left(t_{0}\right)}^{\gamma_{i}^{0}} \omega(\xi, t, \varepsilon \delta \mu) d \xi= & \int_{\gamma_{i}\left(t_{0}\right)}^{\gamma_{i}^{0}} Y(\xi, t) \beta_{i}(\xi) d \xi \\
& -\int_{\gamma_{i}\left(t_{0}\right)}^{\gamma_{i}^{0}} Y(\xi, t) \widetilde{f}_{x_{i}}[\xi] \Delta x\left(\tau_{i}(\xi)\right) d \xi+o(t, \varepsilon \delta \mu),
\end{aligned}
$$

где

$$
\begin{aligned}
& \beta_{i}(\xi)= \widetilde{f}\left(\xi, \widetilde{x}\left(\tau_{1}(\xi)\right)+\Delta x\left(\tau_{1}(\xi)\right), \ldots, \widetilde{x}\left(\tau_{i}(\xi)\right)\right. \\
&\left.+\Delta x\left(\tau_{i}(\xi)\right), \varphi\left(\tau_{i+1}(\xi)\right), \ldots, \varphi\left(\tau_{s}(\xi)\right)\right) \\
&-\widetilde{f}\left(\xi, \widetilde{x}\left(\tau_{1}(\xi)\right), \ldots, \widetilde{x}\left(\tau_{i-1}(\xi)\right), \widetilde{\varphi}\left(\tau_{i}(\xi)\right), \ldots, \widetilde{\varphi}\left(\tau_{s}(\xi)\right)\right), \\
& o(t, \varepsilon \delta \mu)=-\sum_{j=1}^{i-1} \int_{\gamma_{i}\left(t_{0}\right)}^{\gamma_{i}^{0}} Y(\xi, t) \tilde{f}_{x_{j}}[\xi] \Delta x\left(\tau_{j}(\xi)\right) d \xi \\
&-\varepsilon \sum_{j=i+1}^{s} \int_{\gamma_{i}\left(t_{0}\right)}^{\gamma_{i}^{0}} Y(\xi, t) \tilde{f}_{x_{j}}[\xi] \delta \varphi\left(\tau_{j}(\xi)\right) d \xi .
\end{aligned}
$$

Ясно, что

$$
\begin{aligned}
\int_{\gamma_{i}\left(t_{0}\right)}^{\gamma_{i}^{0}} Y(\xi, t) \beta_{i}(\xi) d \xi & =\int_{\gamma_{i}\left(t_{0}\right)}^{\gamma_{i}^{0}} Y(\xi, t)\left(\beta_{i}(\xi)-f_{i}^{-}\right) d \xi+\int_{\gamma_{i}\left(t_{0}\right)}^{\gamma_{i}^{0}} Y(\xi, t) f_{i}^{-} d \xi \\
& =\alpha_{5}(t, \varepsilon \delta \mu)+\alpha_{6}(t, \varepsilon \delta \mu) .
\end{aligned}
$$

Далее, при $\xi \in\left[\gamma_{i}\left(t_{0}\right), \gamma_{i}^{0}\right] \tau_{j}(\xi) \geqslant \widetilde{t}_{0}, j=1, \ldots, i-1, i=p+1, \ldots, s$, поэтому

$$
\lim _{\varepsilon \rightarrow 0}\left(\widetilde{x}\left(\tau_{j}(\xi)\right)+\Delta x\left(\tau_{j}(\xi)\right)\right)=\lim _{\xi \rightarrow \gamma_{i}^{0}} \widetilde{x}\left(\tau_{j}(\xi)\right)=\widetilde{x}\left(\tau_{j}\left(\gamma_{i}^{0}\right)\right), \quad j=1, \ldots, i-1
$$

При $\xi \in\left[\gamma_{i}\left(t_{0}\right), \gamma_{i}^{0}\right] \tau_{i}(\xi) \in\left[t_{0}, \widetilde{t}_{0}\right]$, поэтому

$\widetilde{x}\left(\tau_{i}(\xi)\right)+\Delta x\left(\tau_{i}(\xi)\right)=x\left(\tau_{i}(\xi), \widetilde{\mu}+\varepsilon \delta \mu\right)=y\left(\tau_{i}(\xi), \widetilde{\mu}+\varepsilon \delta \mu\right)=\widetilde{y}\left(\tau_{i}(\xi)\right)+\Delta y\left(\tau_{i}(\xi)\right)$

(см. $(2.7))$. 
Таким образом, учитьвая непрерывность функции $\widetilde{y}(t), t \in\left[\widetilde{t_{0}}-\delta_{2}, \widetilde{t}_{1}+\delta_{2}\right]$, равенство $(2.8)$ и условие $\widetilde{y}\left(\widetilde{t}_{0}\right)=\widetilde{x}_{0}$, будем иметь

$$
\lim _{\varepsilon \rightarrow 0}\left(\widetilde{x}\left(\tau_{i}(\xi)\right)+\Delta x\left(\tau_{i}(\xi)\right)\right)=\lim _{\xi \rightarrow \gamma_{i}} \widetilde{y}\left(\tau_{i}(\xi)\right)=\widetilde{x}_{0} .
$$

Принимая во внимание соотношения, полученные вьше, можно заключить, что при $i=p+1, \ldots, s, \xi \in\left[\gamma_{i}\left(t_{0}\right), \gamma_{i}^{0}\right]$

$$
\begin{aligned}
& \lim _{\varepsilon \rightarrow 0}\left(\xi, \widetilde{x}\left(\tau_{1}(\xi)\right)+\Delta x\left(\tau_{1}(\xi)\right), \ldots, \widetilde{x}\left(\tau_{i}(\xi)\right)\right. \\
& \left.\quad+\Delta x\left(\tau_{i}(\xi)\right), \varphi\left(\tau_{i+1}(\xi)\right), \ldots, \varphi\left(\tau_{s}(\xi)\right)\right)=\sigma_{i} .
\end{aligned}
$$

С другой стороны,

$\lim _{\varepsilon \rightarrow 0}\left(\xi, \widetilde{x}\left(\tau_{1}(\xi)\right), \ldots, \widetilde{x}\left(\tau_{i-1}(\xi)\right), \widetilde{\varphi}\left(\tau_{i}(\xi)\right), \ldots, \widetilde{\varphi}\left(\tau_{s}(\xi)\right)\right)=\sigma_{i}^{0}, \quad i=p+1, \ldots, s$.

Итак,

$$
\lim _{\varepsilon \rightarrow 0} \sup _{\xi \in\left[\gamma_{i}\left(t_{0}\right), \gamma_{i}^{0}\right]}\left|\beta_{i}(\xi)-f_{i}^{-}\right|=0
$$

равномерно по $\delta \mu \in V^{-}$.

Функция $Y(\xi, t)$ непрерьвна на $\left[\gamma_{i}\left(t_{0}\right), \gamma_{i}^{0}\right] \times\left[\widetilde{t}_{1}-\delta_{2}, \widetilde{t}_{1}+\delta_{2}\right] \subset$ П и, кроме того,

$$
\gamma_{i}^{0}-\gamma_{i}\left(t_{0}\right)=-\varepsilon \dot{\gamma}_{i}^{-} \delta t_{0}+o(\varepsilon \delta \mu)
$$

Следовательно, $\alpha_{5}(t, \varepsilon \delta \mu)$ имеет порядок $o(t, \varepsilon \delta \mu)$, а $\alpha_{6}(t, \varepsilon \delta \mu)$ имеет вид

$$
\alpha_{6}(t, \varepsilon \delta \mu)=-\varepsilon Y\left(\gamma_{i}^{0}, t\right) f_{i}^{-} \dot{\gamma}_{i}^{-} \delta t_{0}+o(t, \varepsilon \delta \mu) .
$$

Итак,

$$
\begin{aligned}
\alpha_{2}(t, \varepsilon \delta \mu)= & -\varepsilon \sum_{i=p+1}^{s} Y\left(\gamma_{i}^{0}, t\right) f_{i}^{-} \dot{\gamma}_{i}^{-} \delta t_{0} \\
& -\sum_{i=p+1}^{s} \int_{\gamma_{i}\left(t_{0}\right)}^{\gamma_{i}^{0}} Y(\xi, t) \widetilde{f}_{x_{i}}[\xi] \Delta x\left(\tau_{i}(\xi)\right) d \xi+o(t, \varepsilon \delta \mu) .
\end{aligned}
$$

Аналогичньм способом лишь с незначительньми изменениями доказываются равенства

$$
\alpha_{i}(t, \varepsilon \delta \mu)=o(t, \varepsilon \delta \mu), \quad i=3,4
$$

(см. (3.12)).

Теперь выпишем для $h_{1}\left(t, \widetilde{t}_{0}, \varepsilon \delta \mu\right)$ окончательную формулу

$$
\begin{aligned}
h_{1}\left(t, \widetilde{t}_{0}, \varepsilon \delta \mu\right)= & -\varepsilon \sum_{i=p+1}^{s} Y\left(\gamma_{i}^{0}, t\right) f_{i}^{-} \dot{\gamma}_{i}^{-} \delta t_{0} \\
& -\sum_{i=p+1}^{s} \int_{\gamma_{i}\left(t_{0}\right)}^{\gamma_{i}} Y(\xi, t) \widetilde{f}_{x_{i}}[\xi] \Delta x\left(\tau_{i}(\xi)\right) d \xi+o(t, \varepsilon \delta \mu)
\end{aligned}
$$

(см. (3.11)). 
Наконец, оценим $h_{2}\left(t, \widetilde{t}_{0}, \varepsilon \delta \mu\right)$. Имеем

$$
\begin{aligned}
\left|h_{2}\left(t, \widetilde{t}_{0}, \varepsilon \delta \mu\right)\right| & \leqslant \varepsilon \alpha\|Y\| \sum_{i=1}^{k} \sum_{j=1}^{s} \int_{\tilde{t}_{0}}^{\widetilde{t}_{1}+\delta_{2}} L_{\delta f_{i}, K_{1}}(t)\left|\Delta x\left(\tau_{j}(t)\right)\right| d t \\
& =\varepsilon \alpha\|Y\| \sum_{i=1}^{k} \sum_{j=1}^{s} \sigma_{i j}\left(\widetilde{t}_{0}, \widetilde{t}_{1}+\delta_{2} ; \varepsilon \delta \mu\right)
\end{aligned}
$$

(см. (2.6)).

Докажем, что

$$
\lim _{\varepsilon \rightarrow 0} \sigma_{i j}\left(\widetilde{t_{0}}, \widetilde{t}_{1}+\delta_{2} ; \varepsilon \delta \mu\right)=0, \quad i=1, \ldots, k, \quad j=1, \ldots, s,
$$

равномерно по $\delta \mu \in V^{-}$.

Пусть $j \in\{1, \ldots, p\}$, тогда $\tau_{j}(t) \geqslant \widetilde{t}_{0}$ при $t \in\left[\widetilde{t}_{0}, \widetilde{t}_{1}+\delta_{2}\right]$. Поэтому

$$
\left|\Delta x\left(\tau_{j}(t)\right)\right| \leqslant O(\varepsilon \delta \mu), \quad t \in\left[\widetilde{t_{0}}, \widetilde{t}_{1}+\delta_{1}\right] .
$$

Следовательно, в этом случае равенство (3.15) доказано.

Пусть $j \in\{p+1, \ldots, s$,$\} . Тогда \tau_{j}(t)<t_{0}$ при $t \in\left[\widetilde{t}_{0}, \gamma_{j}\left(t_{0}\right)\right), \tau_{j}(t) \in\left[t_{0}, \widetilde{t}_{0}\right]$ при $t \in\left[\gamma_{j}\left(t_{0}\right), \gamma_{j}^{0}\right], \tau_{j}(t)>\widetilde{t}_{0}$ при $t>\gamma_{j}^{0}$. Поэтому

$$
\left|\Delta x\left(\tau_{j}(t)\right)\right|=\varepsilon \delta \varphi\left(\tau_{j}(t)\right), \quad t \in\left[\widetilde{t}_{0}, \gamma_{j}\left(t_{0}\right)\right), \quad\left|\Delta x\left(\tau_{j}(t)\right)\right| \leqslant O(\varepsilon \delta \mu), \quad t \in\left[\gamma_{j}^{0}, \widetilde{t}_{1}+\delta_{2}\right] .
$$

Легко видеть, что $\gamma_{j}\left(t_{0}\right)-\gamma_{j}^{0} \rightarrow 0$ при $\varepsilon \rightarrow 0$, а функция $\Delta x(t)$ на интервале $\left[\tau_{0}, \widetilde{t_{1}}+\delta_{2}\right]$ ограничена. Кроме того,

$\sigma_{i j}\left(\widetilde{t_{0}}, \widetilde{t_{1}}+\delta_{2} ; \varepsilon \delta \mu\right)=\sigma_{i j}\left(\widetilde{t_{0}}, \gamma_{j}\left(t_{0}\right) ; \varepsilon \delta \mu\right)+\sigma_{i j}\left(\gamma_{j}\left(t_{0}\right), \gamma_{j} ; \varepsilon \delta \mu\right)+\sigma_{i j}\left(\gamma_{j}, \widetilde{t}_{1}+\delta_{2} ; \varepsilon \delta \mu\right)$.

Все слагаемые последнего равенства в силу вышеприведенных рассуждений стремятся к нулю при $\varepsilon \rightarrow 0$ равномерно по $\delta \mu \in V^{-}$. Равенство (3.15) доказано.

Таким образом,

$$
h_{2}\left(t, \widetilde{t_{0}}, \varepsilon \delta \mu\right)=o(t, \varepsilon \delta \mu)
$$

Из (3.7), принимая во внимание (3.9), (3.10), (3.13), (3.16), получаем (1.5), где $\delta x(t, \delta \mu)$ имеет вид (1.6).

\section{§4. Доказательство теоремы 1.2}

Пусть $r_{1}=\widetilde{t}_{0}, r_{2}=\widetilde{t}_{1}$. Тогда для произвольного $(\varepsilon, \delta \mu) \in\left[0, \varepsilon_{1}\right] \times V^{+}$решение $y(t, \widetilde{\mu}+\varepsilon \delta \mu)$ определено на $\left[\widetilde{t}_{1}-\delta_{1}, \widetilde{t}_{1}+\delta_{1}\right]$, а решение $x(t, \widetilde{\mu}+\varepsilon \delta \mu)$ определено на $\left[\tau_{0}, \widetilde{t}_{1}+\delta_{1}\right]$, при этом

$$
y(t, \tilde{\mu}+\varepsilon \delta \mu)=x(t, \widetilde{\mu}+\varepsilon \delta \mu), \quad t \in\left[t_{0}, \widetilde{t}_{1}+\delta_{1}\right]
$$

(см. леммы 2.1, 2.2, (2.4)). 
Таким образом (см. (1.4), (2.7)),

$$
\Delta x(t)= \begin{cases}\varepsilon \delta \varphi(t), & t \in\left[\tau_{0}, \widetilde{t}_{0}\right) \\ \varphi(t)-\widetilde{x}(t), & t \in\left[\widetilde{t}_{0}, t_{0}\right) ; \\ \Delta y(t), & t \in\left[t_{0}, \widetilde{t}_{1}+\delta_{1}\right) .\end{cases}
$$

Пусть числа $\delta_{2} \in\left(0, \delta_{1}\right]$ и $\varepsilon_{2} \in\left(0, \varepsilon_{1}\right]$, сушествование которых доказывается в лемме 2.5 , настолько малы, что для произвольного элемента $(\varepsilon, \delta \mu) \in\left(0, \varepsilon_{2}\right] \times V^{+}$ вьполнено неравенство

$$
\gamma_{s}\left(t_{0}\right)<\widetilde{t}_{1}-\delta_{2}
$$

В силу леммы 2.5 с учетом (4.1) будем иметь

$$
\begin{gathered}
|\Delta x(t)| \leqslant O(\varepsilon \delta \mu) \quad \forall(t, \varepsilon \delta \mu) \in\left[t_{0}, \widetilde{t}_{1}+\delta_{2}\right] \times\left[0, \varepsilon_{2}\right] \times V^{+} \\
\Delta x\left(t_{0}\right)=\varepsilon\left(\delta x_{0}-f_{p}^{+} \delta t_{0}\right)+o(\varepsilon \delta \mu) .
\end{gathered}
$$

Функция $\Delta x(t)$ на интервале $\left[t_{0}, \widetilde{t}_{1}+\delta_{2}\right]$ удовлетворяет уравнению (3.4). Поэтому согласно формуле Коши ее можно представить в виде

$$
\begin{gathered}
\Delta x(t)=Y\left(t_{0}, t\right) \Delta x\left(t_{0}\right)+\varepsilon \int_{t_{0}}^{t} Y(\xi, t) \delta f[\xi] d \xi+\sum_{i=0}^{2} h_{i}\left(t, t_{0}, \varepsilon \delta \mu\right), \\
t \in\left[t_{0}, \widetilde{t_{1}}+\delta_{2}\right]
\end{gathered}
$$

где

$$
h_{0}\left(t, t_{0}, \varepsilon \delta \mu\right)=\sum_{i=1}^{s} \int_{\tau_{i}\left(t_{0}\right)}^{t_{0}} Y\left(\gamma_{i}(\xi), t\right) \widetilde{f}_{x_{i}}\left[\gamma_{i}(\xi)\right] \dot{\gamma}_{i}(\xi) \Delta x(\xi) d \xi,
$$

а $h_{i}\left(t, t_{0}, \varepsilon \delta \mu\right), i=1,2$, имеют соответственно вид (3.8).

В силу леммы 3.4 [5] функция $Y(\xi, t)$ непрерьвна на множестве

$$
\left[\widetilde{t}_{0}, \tau_{s}\left(\widetilde{t}_{1}-\delta_{2}\right)\right] \times\left[\widetilde{t_{1}}-\delta_{2}, \widetilde{t}_{1}+\delta_{2}\right]
$$

Ясно, что $t_{0} \in\left[\widetilde{t_{0}}, \tau_{s}\left(\widetilde{t_{1}}-\delta_{2}\right)\right]$, поэтому

$$
Y\left(t_{0}, t\right) \Delta x\left(t_{0}\right)=\varepsilon Y\left(\widetilde{t_{0}}, t\right)\left(\delta x_{0}-f_{p}^{+} \delta t_{0}\right)+o(t, \varepsilon \delta \mu)
$$

(см. $(4.3))$. 
Теперь преобразуем (4.5). Имеем

$$
\begin{aligned}
h_{0}\left(t, t_{0}, \varepsilon \delta \mu\right)= & \sum_{i=1}^{p} \int_{\tau_{i}\left(t_{0}\right)}^{t_{0}} Y\left(\gamma_{i}(\xi), t\right) \widetilde{f}_{x_{i}}\left[\gamma_{i}(\xi)\right] \dot{\gamma}_{i}(\xi) \Delta x(\xi) d \xi \\
& +\sum_{i=p+1}^{s}\left(\varepsilon \int_{\tau_{i}\left(t_{0}\right)}^{\widetilde{t}_{0}} Y\left(\gamma_{i}(\xi), t\right) \widetilde{f}_{x_{i}}\left[\gamma_{i}(\xi)\right] \dot{\gamma}_{i}(\xi) \delta \varphi(\xi) d \xi\right. \\
& \left.+\int_{\widetilde{t}_{0}}^{t_{0}} Y\left(\gamma_{i}(\xi), t\right) \widetilde{f}_{x_{i}}\left[\gamma_{i}(\xi)\right] \dot{\gamma}_{i}(\xi) \Delta x(\xi) d \xi\right) \\
= & \sum_{i=1}^{p} \int_{t_{0}}^{\gamma_{i}\left(t_{0}\right)} Y(\xi, t) \widetilde{f}_{x_{i}}[\xi] \Delta x\left(\tau_{i}(\xi)\right) d \xi \\
& +\varepsilon \sum_{i=p+1}^{s} \int_{\tau_{i}\left(\widetilde{t}_{0}\right)}^{\bar{t}_{0}} Y\left(\gamma_{i}(\xi), t\right) \tilde{f}_{x_{i}}\left[\gamma_{i}(\xi)\right] \dot{\gamma}_{i}(\xi) \delta \varphi(\xi) d \xi \\
& +\sum_{i=p+1}^{s} \int_{\gamma_{i}^{0}}^{\gamma_{i}\left(t_{0}\right)} Y(\xi, t) \widetilde{f}_{x_{i}}[\xi] \Delta x\left(\tau_{i}(\xi) d \xi+o(t, \varepsilon \delta \mu) .\right.
\end{aligned}
$$

После элементарных преобразований легко получаем следуюшее равенство:

$$
\begin{aligned}
& \sum_{i=1}^{p} \int_{t_{0}}^{\gamma_{i}\left(t_{0}\right)} Y(\xi, t) \widetilde{f}_{x_{i}}[\xi] \Delta x\left(\tau_{i}(\xi)\right) d \xi=\sum_{i=1}^{p} \sum_{j=0}^{i-1} \int_{\gamma_{j}\left(t_{0}\right)}^{\gamma_{j+1}\left(t_{0}\right)} Y(\xi, t) \widetilde{f}_{x_{i}}[\xi] \Delta x\left(\tau_{i}(\xi)\right) d \xi \\
& \quad=\sum_{i=0}^{p-1} \sum_{j=i+1}^{p} \int_{\gamma_{i}\left(t_{0}\right)}^{\gamma_{i+1}\left(t_{0}\right)} Y(\xi, t) \widetilde{f}_{x_{j}}[\xi] \Delta x\left(\tau_{j}(\xi)\right) d \xi, \quad \gamma_{0}\left(t_{0}\right)=t_{0}
\end{aligned}
$$

Согласно неравенствам (2.30) $h_{1}\left(t, t_{0}, \varepsilon \delta \mu\right)$ при $t \in\left[\widetilde{t}_{1}-\delta_{2}, \widetilde{t}_{1}+\delta_{2}\right]$ можно представить в виде

$$
h_{1}\left(t, t_{0}, \varepsilon \delta \mu\right)=\sum_{k=1}^{5} \beta_{k}(t, \varepsilon \delta \mu),
$$

где

$$
\begin{aligned}
\beta_{1}(t, \varepsilon \delta \mu) & =\sum_{i=0}^{p-1} \int_{\gamma_{i}\left(t_{0}\right)}^{\gamma_{i+1}\left(t_{0}\right)} \omega(\xi, t, \varepsilon \delta \mu) d \xi \\
\beta_{2}(t, \varepsilon \delta \mu) & =\int_{\gamma_{p}\left(t_{0}\right)}^{\gamma_{p+1}^{0}} \omega(\xi, t, \varepsilon \delta \mu) d \xi \\
\beta_{3}(t, \varepsilon \delta \mu) & =\sum_{i=p+1}^{s} \int_{\gamma_{i}^{0}}^{\gamma_{i}\left(t_{0}\right)} \omega(\xi, t, \varepsilon \delta \mu) d \xi, \\
\beta_{4}(t, \varepsilon \delta \mu) & =\sum_{i=p+1}^{s-1} \int_{\gamma_{i}\left(t_{0}\right)}^{\gamma_{i+1}^{0}} \omega(\xi, t, \varepsilon \delta \mu) d \xi, \\
\beta_{5}(t, \varepsilon \delta \mu) & =\int_{\gamma_{s}\left(t_{0}\right)}^{t} \omega(\xi, t, \varepsilon \delta \mu) d \xi \\
\omega(\xi, t, \varepsilon \delta \mu) & =Y(\xi, t) R_{1}(\xi, \varepsilon \delta \mu) .
\end{aligned}
$$


Для $\beta_{1}(t, \varepsilon \delta \mu)$ будем иметь

$$
\begin{aligned}
& \beta_{1}(t, \varepsilon \delta \mu)=\sum_{i=0}^{p-1} \int_{\gamma_{i}\left(t_{0}\right)}^{\gamma_{i+1}\left(t_{0}\right)} Y(\xi, t)\left(\widetilde { f } \left(\xi, \widetilde{x}\left(\tau_{1}(\xi)\right)\right.\right. \\
& \left.\quad+\Delta x\left(\tau_{1}(\xi)\right), \ldots, \widetilde{x}\left(\tau_{i}(\xi)\right)+\Delta x\left(\tau_{i}(\xi)\right), \varphi\left(\tau_{i+1}(\xi)\right), \ldots, \varphi\left(\tau_{s}(\xi)\right)\right) \\
& \left.\quad-\widetilde{f}\left(\xi, \widetilde{x}\left(\tau_{1}(\xi)\right), \ldots, \widetilde{x}\left(\tau_{p}(\xi)\right), \widetilde{\varphi}\left(\tau_{p+1}(\xi)\right), \ldots, \widetilde{\varphi}\left(\tau_{s}(\xi)\right)\right)\right) d \xi \\
& \quad-\sum_{i=0}^{p-1} \int_{\gamma_{i}\left(t_{0}\right)}^{\gamma_{i+1}\left(t_{0}\right)} Y(\xi, t) \sum_{j=1}^{s} \widetilde{f}_{x_{i}}[\xi] \Delta x\left(\tau_{j}(\xi)\right) d \xi=\beta_{11}(t, \varepsilon \delta \mu)-\beta_{12}(t, \varepsilon \delta \mu) .(4.10)
\end{aligned}
$$

При $\xi \in\left[\gamma_{i}\left(t_{0}\right), \gamma_{i+1}\left(t_{0}\right)\right]$

$$
\tau_{j}(\xi) \geqslant t_{0}, j=1, \ldots, i, \quad \tau_{j}(\xi)<t_{0}, j=i, \ldots, p, \quad \tau_{j}(\xi)<\widetilde{t}_{0}, j=p+1, \ldots, s
$$

поэтому

$$
\begin{aligned}
\left|\Delta x\left(\tau_{j}(\xi)\right)\right| & \leqslant O(\varepsilon \delta \mu), & & j=1, \ldots, i, \\
\Delta x\left(\tau_{j}(\xi)\right) & =\varepsilon \delta \varphi\left(\tau_{j}(\xi)\right), & & j=p+1, \ldots, s
\end{aligned}
$$

(см. (4.1), (4.3)).

$$
\begin{aligned}
& \text { Для каждого } i=0, \ldots, p-1 \gamma_{i+1}\left(t_{0}\right)-\gamma_{i}\left(t_{0}\right) \rightarrow 0 \text { при } \varepsilon \rightarrow 0 \text {. Следовательно, } \\
& \beta_{12}(t, \varepsilon \delta \mu)=\sum_{i=0}^{p-1} \sum_{j=i+1}^{p} \int_{\gamma_{i}\left(t_{0}\right)}^{\gamma_{i+1}\left(t_{0}\right)} Y(\xi, t) \widetilde{f}_{x_{i}}[\xi] \Delta x\left(\tau_{j}(\xi)\right) d \xi+o(t, \varepsilon \delta \mu) .
\end{aligned}
$$

Далее,

$$
\begin{aligned}
& \lim _{\varepsilon \rightarrow 0} \sup _{\xi \in\left[\gamma_{i}\left(t_{0}\right), \gamma_{i+1}\left(t_{0}\right)\right]} \mid \widetilde{f}\left(\xi, \widetilde{x}\left(\tau_{1}(\xi)\right)+\Delta x\left(\tau_{1}(\xi)\right), \ldots, \widetilde{x}\left(\tau_{i}(\xi)\right)\right. \\
& \left.\quad+\Delta x\left(\tau_{i}(\xi)\right), \varphi\left(\tau_{i+1}(\xi)\right), \ldots, \varphi\left(\tau_{s}(\xi)\right)\right)-f_{i}^{-}+f_{p}^{+} \\
& \quad-\widetilde{f}\left(\xi, \widetilde{x}\left(\tau_{1}(\xi)\right), \ldots, \widetilde{x}\left(\tau_{p}(\xi)\right), \widetilde{\varphi}\left(\tau_{p+1}(\xi)\right), \ldots, \widetilde{\varphi}\left(\tau_{s}(\xi)\right)\right) \mid=0, \quad i=0, \ldots, p-1,
\end{aligned}
$$

равномерно по $\delta \mu \in V^{+}$.

Из свойств функций $Y(\xi, t), \gamma_{i}(t), i=1, \ldots, p$, непосредственно следует

$$
\lim _{\varepsilon \rightarrow 0} \sup _{\xi \in\left[\gamma_{i}\left(t_{0}\right), \gamma_{i+1}\left(t_{0}\right)\right]}\left|Y(\xi, t)-Y\left(\widetilde{t_{0}}, t\right)\right|=0, \quad i=0, \ldots, p-1,
$$

равномерно по $t \in\left[\widetilde{t}_{1}-\delta_{2}, \widetilde{t}_{1}+\delta_{2}\right]$,

$$
\gamma_{i+1}\left(t_{0}\right)-\gamma_{i}\left(t_{0}\right)=\varepsilon\left(\dot{\gamma}_{i+1}^{+}-\dot{\gamma}_{i}^{+}\right) \delta t_{0}+o(\varepsilon \delta \mu), \quad i=0, \ldots, p-1, \quad \dot{\gamma}_{0}=1
$$


Эти условия для $\beta_{11}(t, \varepsilon \delta \mu)$ дают

$$
\beta_{11}(t, \varepsilon \delta \mu)=\varepsilon Y\left(\widetilde{t}_{0}, t\right) \sum_{i=0}^{p}\left(f_{i}^{+}-f_{p}^{+}\right)\left(\dot{\gamma}_{i+1}^{+}-\dot{\gamma}_{i}^{+}\right) \delta t_{0}+o(t, \varepsilon \delta \mu) .
$$

Из (4.10) с учетом (4.11), (4.12) вытекает

$$
\begin{aligned}
& \beta_{1}(t, \varepsilon \delta \mu)=\varepsilon Y\left(\widetilde{t}_{0}, t\right)\left(\sum_{i=0}^{p}\left(\widehat{\gamma}_{i+1}^{+}-\widehat{\gamma}_{i}^{+}\right) f_{i}^{+}+f_{p}^{+}\right) \delta t_{0} \\
& -\sum_{i=0}^{p-1} \sum_{j=i+1}^{p} \int_{\gamma_{i}\left(t_{0}\right)}^{\gamma_{i+1}\left(t_{0}\right)} Y(\xi, t) \widetilde{f}_{x_{j}}[\xi] \Delta x\left(\tau_{j}(\xi)\right) d \xi+o(t, \varepsilon \delta \mu) .
\end{aligned}
$$

Далее,

$$
\begin{aligned}
\beta_{2}(t, \varepsilon \delta \mu)= & \int_{\gamma_{p}\left(t_{0}\right)}^{\gamma_{p+1}^{0}} Y(\xi, t)\left(\widetilde { f } \left(\xi, \widetilde{x}\left(\tau_{i}(\xi)\right)\right.\right. \\
& \left.+\Delta x\left(\tau_{1}(\xi)\right), \ldots, \widetilde{x}\left(\tau_{p}(\xi)\right)+\Delta x\left(\tau_{p}(\xi)\right), \varphi\left(\tau_{p+1}(\xi)\right), \ldots, \varphi\left(\tau_{s}(\xi)\right)\right) \\
& -\widetilde{f}\left(\xi, \widetilde{x}\left(\tau_{1}(\xi)\right), \ldots, \widetilde{x}\left(\tau_{p}(\xi)\right), \widetilde{\varphi}\left(\tau_{p+1}(\xi)\right), \ldots, \widetilde{\varphi}\left(\tau_{s}(\xi)\right)\right) \\
& \left.-\sum_{j=1}^{p} \widetilde{f}_{x_{j}}[\xi] \Delta x\left(\tau_{j}(\xi)\right)-\varepsilon \sum_{j=p+1}^{s} \widetilde{f}_{x_{j}}[\xi] \delta \varphi\left(\tau_{j}(\xi)\right)\right) d \xi .
\end{aligned}
$$

Стандартным способом (см. (3.12)) доказывается, что

$$
\left|\beta_{2}(t, \varepsilon \delta \mu)\right| \leqslant\|Y\|\left(O(\varepsilon \delta \mu) \sum_{i=1}^{p} \sigma_{i}\left(t_{0}, \varepsilon \delta \mu\right)+\varepsilon \alpha \sum_{i=p+1}^{s} \sigma_{i}\left(t_{0}, \varepsilon \delta \mu\right)\right),
$$

где

$$
\sigma_{i}\left(t_{0}, \varepsilon \delta \mu\right)=\int_{\gamma_{p}\left(t_{0}\right)}^{\gamma_{p+1}^{0}}\left(\int_{0}^{1}\left|\widetilde{f}_{x_{i}}\left(t, \widetilde{x}\left(\tau_{1}(t)\right)+\xi \Delta x\left(\tau_{1}(t)\right), \ldots\right)-\widetilde{f}_{x_{i}}[t]\right| d \xi\right) d t .
$$

При $t \in\left[\gamma_{p}\left(t_{0}\right), \gamma_{p+1}^{0}\right] \tau_{j}(t) \geqslant t_{0}, j=1, \ldots, p$. Поэтому (см. (4.1))

$$
\Delta x\left(\tau_{j}(t)\right)=\Delta y\left(\tau_{j}(t)\right) .
$$

Следовательно,

$$
\begin{aligned}
& \sigma_{i}\left(t_{0}, \varepsilon \delta \mu\right)=\int_{\gamma_{p}\left(t_{0}\right)}^{\gamma_{p+1}^{0}}\left(\int_{0}^{1} \mid \widetilde{f}_{x_{i}}\left(t, \widetilde{x}\left(\tau_{1}(t)\right)\right.\right. \\
& \quad+\xi \Delta y\left(\tau_{1}(t)\right), \ldots, \widetilde{x}\left(\tau_{p}(t)\right)+\xi \Delta y\left(\tau_{p}(t)\right), \widetilde{\varphi}\left(\tau_{p+1}(t)\right) \\
& \left.\quad+\xi \varepsilon \delta \varphi\left(\tau_{p+1}(t)\right), \ldots, \widetilde{\varphi}\left(\tau_{s}(t)\right)+\xi \varepsilon \delta \varphi\left(\tau_{s}(t)\right)\right) \\
& \left.\quad-\widetilde{f}_{x_{i}}\left(t, \widetilde{x}\left(\tau_{1}(t)\right), \ldots, \widetilde{x}\left(\tau_{p}(t)\right), \widetilde{\varphi}\left(\tau_{p+1}(t)\right), \ldots, \widetilde{\varphi}\left(\tau_{s}(t)\right)\right) \mid d \xi\right) d t
\end{aligned}
$$


Отсюда, как легко видеть (см. (2.8)),

$$
\lim _{\varepsilon \rightarrow 0} \sigma_{i}\left(t_{0}, \varepsilon \delta \mu\right)=0
$$

равномерно по $\delta \mu \in V^{+}$.

Таким образом,

$$
\beta_{2}(t, \varepsilon \delta \mu)=o(t, \varepsilon \delta \mu)
$$

(см. (4.14)).

Преобразуем остальные слагаемые выражения (4.9). Имеем

$$
\begin{aligned}
& \beta_{3}(t, \varepsilon \delta \mu)=\sum_{i=p+1}^{s} \int_{\gamma_{i}^{0}}^{\gamma_{i}\left(t_{0}\right)} Y(\xi, t)\left(\widetilde { f } \left(\xi, \widetilde{x}\left(\tau_{1}(\xi)\right)\right.\right. \\
& \left.\quad+\Delta x\left(\tau_{1}(\xi)\right), \ldots, \widetilde{x}\left(\tau_{i-1}(\xi)\right)+\Delta x\left(\tau_{i-1}(\xi)\right), \varphi\left(\tau_{i}(\xi)\right), \ldots, \varphi\left(\tau_{s}(\xi)\right)\right) \\
& \left.\quad-\widetilde{f}\left(\xi, \widetilde{x}\left(\tau_{1}(\xi)\right), \ldots, \widetilde{x}\left(\tau_{i}(\xi)\right), \widetilde{\varphi}\left(\tau_{i+1}(\xi)\right), \ldots, \widetilde{\varphi}\left(\tau_{s}(\xi)\right)\right)\right) d \xi \\
& \quad-\sum_{i=p+1}^{s}\left(\sum_{j=1}^{i-1} \int_{\gamma_{i}^{0}}^{\gamma_{i}\left(t_{0}\right)} Y(\xi, t) \widetilde{f}_{x_{j}}[\xi] \Delta x\left(\tau_{j}(\xi)\right) d \xi\right. \\
& \left.\quad+\int_{\gamma_{i}^{0}}^{\gamma_{i}\left(t_{0}\right)} Y(\xi, t) \widetilde{f}_{x_{i}}[\xi] \Delta x\left(\tau_{i}(\xi)\right) d \xi+\varepsilon \sum_{j=i+1}^{s} \int_{\gamma_{i}^{0}}^{\gamma_{i}\left(t_{0}\right)} Y(\xi, t) \tilde{f}_{x_{j}}[\xi] \delta \varphi\left(\tau_{j}(\xi)\right) d \xi\right) .
\end{aligned}
$$

Согласно условию 1.5) будем иметь

$$
\begin{gathered}
\lim _{\varepsilon \rightarrow 0} \sup _{\xi \in\left[\gamma_{i}^{0}, \gamma_{i}\left(t_{0}\right)\right]} \mid \widetilde{f}\left(\xi \widetilde{x}\left(\tau_{1}(\xi)\right)+\Delta x\left(\tau_{1}(\xi)\right), \ldots, \widetilde{x}\left(\tau_{i-1}(\xi)\right), \varphi\left(\tau_{i}(\xi)\right), \ldots, \varphi\left(\tau_{s}(\xi)\right)\right) \\
-\widetilde{f}\left(\xi, \widetilde{x}\left(\tau_{1}(\xi)\right), \ldots, \widetilde{x}\left(\tau_{i}(\xi)\right), \widetilde{\varphi}\left(\tau_{i+1}(\xi)\right), \ldots, \widetilde{\varphi}\left(\tau_{s}(\xi)\right)\right)+f_{i}^{+} \mid=0 \\
i=p+1, \ldots, s
\end{gathered}
$$

равномерно по $\delta \mu \in V^{+}$.

Далее,

$$
\begin{aligned}
& \left|\Delta x\left(\tau_{j}(\xi)\right)\right| \leqslant O(\varepsilon \delta \mu), \quad j=1, \ldots, i-1, \quad \xi \in\left[\gamma_{i}^{0}, \gamma_{i}\left(t_{0}\right)\right] \\
& \lim _{\varepsilon \rightarrow 0} \sup _{\xi \in\left[\gamma_{i}^{0}, \gamma_{i}\left(t_{0}\right)\right]}\left|Y(\xi, t)-Y\left(\gamma_{i}, t\right)\right|=0, \quad i=p+1, \ldots, s
\end{aligned}
$$

равномерно по $t \in\left[\widetilde{t}_{1}-\delta_{2}, \widetilde{t}_{1}+\delta_{2}\right]$.

В силу этих соотношений получаем

$$
\begin{aligned}
\beta_{3}(t, \varepsilon \delta \mu)= & -\varepsilon \sum_{i=p+1}^{s} Y\left(\gamma_{i}^{0}, t\right) f_{i}^{+} \delta t_{0} \\
& -\sum_{i=p+1}^{s} \int_{\gamma_{i}^{0}}^{\gamma_{i}\left(t_{0}\right)} Y(\xi, t) \tilde{f}_{x_{i}}[\xi] \Delta x\left(\tau_{j}(\xi)\right) d \xi+o(t, \varepsilon \delta \mu) .
\end{aligned}
$$


Аналогичным рассуждением (см. (3.12)) доказывается, что

$$
\beta_{i}(t, \varepsilon \delta \mu)=o(t, \varepsilon \delta \mu), \quad i=4,5
$$

В силу соотношений (4.13), (4.15)-(4.17) вьпишем окончательное выражение

$$
\begin{aligned}
h_{1}\left(t, t_{0}, \varepsilon \delta \mu\right)= & \varepsilon\left(Y\left(\widetilde{t}_{0}, t\right) \sum_{i=0}^{p}\left(\widehat{\gamma}_{i+1}^{+}-\widehat{\gamma}_{i}^{+}\right)-\sum_{i=p+1}^{s} Y\left(\gamma_{i}^{0}, t\right) f_{i}^{+}\right) \delta t_{0} \\
& -\sum_{i=0}^{p-1} \sum_{j=i+1}^{p} \int_{\gamma_{i}\left(t_{0}\right)}^{\gamma_{i+1}\left(t_{0}\right)} Y(\xi, t) \widetilde{f}_{x_{i}}[\xi] \Delta x\left(\tau_{j}(\xi)\right) d \xi \\
& -\sum_{i=p+1}^{s} \int_{\gamma_{i}^{0}}^{\gamma_{i}\left(t_{0}\right)} Y(\xi, t) \tilde{f}_{x_{i}}[\xi] \Delta x\left(\tau_{j}(\xi)\right) d \xi+o(t, \varepsilon \delta \mu)
\end{aligned}
$$

(см. (4.9)).

Наконец, оценим $h_{2}\left(t, t_{0}, \varepsilon \delta \mu\right)$. Имеем

$$
\left|h_{2}\left(t, t_{0}, \varepsilon \delta \mu\right)\right| \leqslant \varepsilon \alpha\|Y\| \sum_{i=1}^{k} \sum_{j=1}^{s} \sigma_{i j}\left(t_{0}, \widetilde{t}_{1}+\delta_{2} ; \varepsilon \delta \mu\right)
$$

(см. (3.14)).

Докажем, что

$$
\lim _{\varepsilon \rightarrow 0} \sigma_{i j}\left(t_{0}, \widetilde{t}_{1}+\delta_{2} ; \varepsilon \delta \mu\right)=0, \quad i=1, \ldots, k, \quad j=1, \ldots, s
$$

равномерно по $\delta \mu \in V^{+}$.

Пусть $i \in\{1, \ldots, p\}$, тогда при $t \in\left[\gamma_{i}\left(t_{0}\right), \widetilde{t}_{1}+\delta_{2}\right]$

$$
\left|\Delta x\left(\tau_{i}(t)\right)\right| \leqslant O(\varepsilon \delta \mu)
$$

Легко видеть, что $\gamma_{j}\left(t_{0}\right)-\widetilde{t}_{0} \rightarrow 0$ при $\varepsilon \rightarrow 0$, а функция $\Delta x(t)$ на интервале $\left[\tau, \widetilde{t}_{1}+\delta_{2}\right]$ ограничена. Кроме того,

$$
\sigma_{i j}\left(t_{0}, \widetilde{t}_{1}+\delta_{2} ; \varepsilon \delta \mu\right)=\sigma_{i j}\left(t_{0}, \gamma_{j}\left(t_{0}\right) ; \varepsilon \delta \mu\right)+\sigma_{i j}\left(\gamma_{j}\left(t_{0}\right), \widetilde{t}_{1}+\delta_{2} ; \varepsilon \delta \mu\right) .
$$

Пусть $j \in\{p+1, \ldots, s\}$, тогда

$\Delta x\left(\tau_{j}(t)\right)=\varepsilon \delta \varphi\left(\tau_{j}(t)\right), \quad t \in\left[t_{0}, \gamma_{j}^{0}\right], \quad\left|\Delta x\left(\tau_{j}(t)\right)\right| \leqslant O(\varepsilon \delta \mu), \quad t \in\left[\gamma_{j}\left(t_{0}\right), \widetilde{t}_{1}+\delta_{2}\right]$.

Ясно, что $\gamma_{j}\left(t_{0}\right)-\gamma_{j}^{0} \rightarrow 0$ при $\varepsilon \rightarrow 0$, а $\sigma_{i j}\left(t_{0}, \widetilde{t_{1}} ; \varepsilon \delta \mu\right)$ можно представить в виде суммы

$\sigma_{i j}\left(t_{0}, \widetilde{t}_{1}+\delta_{2} ; \varepsilon \delta \mu\right)=\sigma_{i j}\left(t_{0}, \gamma_{j}^{0} ; \varepsilon \delta \mu\right)+\sigma_{i j}\left(\gamma_{j}^{0}, \gamma_{j}\left(t_{0}\right) ; \varepsilon \delta \mu\right)+\sigma_{i j}\left(\gamma_{j}\left(t_{0}\right), \widetilde{t}_{1}+\delta_{2} ; \varepsilon \delta \mu\right)$. 
Очевидно, что все слагаемые в выражениях (4.20), (4.21) при $\varepsilon \rightarrow 0$ стремятся

к нулю. Следовательно, доказано равенство (4.19), которое в свою очередь дает

$$
h_{2}\left(t, t_{0}, \varepsilon \delta \mu\right)=o(t, \varepsilon \delta \mu) .
$$

В заключение заметим, что при $t \in\left[\widetilde{t}_{1}-\delta_{2}, \widetilde{t}_{1}+\delta_{2}\right]$

$$
\varepsilon \int_{t_{0}}^{t} Y(\xi, t) \delta f[t] d \xi=\varepsilon \int_{\widetilde{t}_{0}}^{t} Y(\xi, t) \delta f[t] d \xi+o(t, \varepsilon \delta \mu) .
$$

Из (4.4), принимая во внимание (4.6)-(4.8), (4.18), (4.22), (4.23), получаем (1.5), где $\delta x(t, \delta \mu)$ имеет вид (1.9).

\section{Список литературы}

1. Понтрягин Л. С., Болтянский В. Г., Гамкрелидзе Р. В., Мищенко Е. Ф. Математическая теория оптимальных процессов. М.: Наука, 1961.

2. Gamkrelidze R. V. On some extremal problems in the theory of differential equation with application to the theory of optimal control // SIAM J. Control Optim. 1965. V. 3. P. 505-527.

3. Харатишвили Г. Л. Оптимальные процессы с запаздьваниями. Тбилиси: Мецниереба, 1966.

4. Ogustoreli N. Time-lag control systems. New York: Academic Press, 1966.

5. Banks H. T. Necessary conditions for control problems with variable time lags // SIAM J. Control Optim. 1968. V. 6. №1. P. 9-47.

6. Гамкрелидзе Р. В., Харатишвили Г. Л. Экстремалњные задачи в линейных топологических пространствах // Изв. АН СССР. Сер. матем. 1969. Т. 33. № 4. С. 781-839.

7. Харатишвили Г. Л., Мачаидзе З. А., Маркозашвили Н. И., Тадумадзе Т. А. Абстрактная вариационная теория и ее применения к оптимальньм задачам с запаздываниями. Тбилиси: Мецниереба 1973.

8. Гамкрелидзе Р. В. Основы оптимального управления. Тбилиси: Изд-во Тбил. гос. ун-та, 1975.

9. Понтрягин Л. С. Обыкновенные дифференциальные уравнения. М.: Наука, 1965.

10. Хейл Дж. Теория функционально-диффференциальных уравнений. М.: Мир, 1984.

11. Kharatishvili G., Tadumadze T., Gorgodze N. Continuous dependence and differentiability of solution with respect to initial data and right-hand side for differential equations with deviating argument // Mem. Differential Equations Math. Phys. 2000. V. 19. P. 3-105.

Институт кибернетики АН Грузии, Тбилиси

Институт прикладной математики им. И.Н. Векуа

Тбилисского государственного университета

E-mail: kharatishvili@cybernet.ge

tamaz_1@hotmail.com

tamaztad@yahoo.com
Поступила в редакцию 17.06 .2003 и 27.10 .2004 